This paper was submitted for publication in IEEE Transactions on Communications on July 11, 2019.

(C2019 IEEE. Personal use of this material is permitted. Permission from IEEE must be obtained for all other uses, in any current or future media, including reprinting/republishing this material for advertising or promotional purposes, creating new collective works, for resale or redistribution to servers or lists, or reuse of any copyrighted component of this work in other works. 


\title{
Rate Analysis of Cell-Free Massive MIMO-NOMA With Three Linear Precoders
}

\author{
Fatemeh Rezaei, Student Member, IEEE, Chintha Tellambura, Fellow, IEEE, Aliakbar Tadaion, Senior \\ Member, IEEE, and Ali Reza Heidarpour, Student Memeber, IEEE
}

\begin{abstract}
Although the hybrid of cell-free (CF) massive multiple-input multiple-output (MIMO) and non-orthogonal multiple access (NOMA) promises massive spectral efficiency gains, the type of precoders employed at the access points (APs) impacts the gains. In this paper, we thus comprehensively evaluate the system performance with maximum ratio transmission (MRT), fullpilot zero-forcing (fpZF) and modified regularized ZF (mRZF) precoders. We derive their closed-form sum rate expressions by considering Rayleigh fading channels, the effects of intra-cluster pilot contamination, inter-cluster interference, and imperfect successive interference cancellation (SIC). Our results reveal that this system supports significantly more users simultaneously at the same coherence interval compared to its OMA equivalent. However, intra-cluster pilot contamination and imperfect SIC degrade the system performance when the number of users is low. Moreover, with perfect SIC, mRZF and fpZF significantly outperform MRT. Also, we show that this system with either mRZF or fpZF precoding outperforms OMA systems with MRT. The analytical findings are verified by numerical results.
\end{abstract}

Index Terms-NOMA, cell-free massive MIMO, MRT, fpZF, modified RZF, achievable sum rate.

\section{INTRODUCTION}

\section{A. Background and Scope}

I NTERNATIONAL mobile telecommunications (IMT)2020 standard is envisioned to support peak data rates up to $10 \mathrm{~Gb} / \mathrm{s}$ for low mobility users and $1 \mathrm{~Gb} / \mathrm{s}$ for high mobility users, $1 \mathrm{~ms}$ over-the-air latency, and a network energy efficiency improvement of $100 \times$ of $4 \mathrm{G}$ networks [1]. These requirements have necessitated the development of new wireless technologies that will offer higher data rates, higher energy efficiency, and ultra low latency.

One such technology is the use of distributed large antenna arrays, which have the ability to increase ergodic sum rate [2], extend coverage [3], and improve energy efficiency [4]. Thus, distributed access points (APs) in each cell of a cellular network have been already studied [2], [5]. The use of distributed APs enables the efficient utilization of spatial resources [6]. However, inter-cell interference is inherent in all cell-centric systems and becomes a major performance limiting factor [7]. To overcome this, while preserving the main benefits of massive MIMO (m-MIMO), cell-free (CF) mMIMO has been proposed [8], [9]. The basic premise of it is that a large number of spatially-distributed APs serve many single-antenna users in the same time-frequency resources [8]. Thus, each user is served by all the APs it can reach, and hence, it does not experience cell boundaries (cell-free). A central processing unit (CPU) coordinates the APs, which are connected to it through a fronthaul network. Locality of the operations of each AP is another key idea, which minimizes the fronthaul overhead. Thus, each AP performs precoding based on only the estimates of the channels from itself to all the users. These downlink channels can be estimated with the help of pilots transmitted by the users in the uplink, thereby exploiting the channel reciprocity inherent in time-division duplexing (TDD). This architecture offers increased macro-diversity and favorable propagation with negligible inter-user interference. And it outperforms the conventional cellular counterparts by exploiting the best of both collocated mMIMO and network MIMO systems [8]-[12].

Precoder design, power control, hardware impairments and other factors for CF mMIMO systems have been investigated. For instance, [8] and [9] investigate conjugate beamforming (CB) (which maximizes the signal gain at the intended user), and zero-forcing (ZF) (which nulls the inter-user interference at the expense of gain losses), and they show that significant spectral efficiency gains are achievable over conventional small-cell systems. The problem of joint power control and load balancing using $\mathrm{ZF}$ and maximum ratio combining (MRC) processing is investigated [13]. How the spectral efficiency is impacted by hardware impairments at both users and APs has also been studied [14]; the key insight is that the negative effects of hardware impairment vanish as the number of APs increases.

Non-orthogonal multiple access (NOMA) also achieves high spectral efficiency gains [15], [16]. It however is a paradigm shift from conventional orthogonal multiple access (OMA) techniques such as time division multiple access and frequency division multiple access, where an orthogonal channel must be created for each different user. In contrast, NOMA serves multiple users simultaneously on each orthogonal channel. This can be done by exploiting channel gain disparities and then performing individual user signal detection via successive interference cancellation (SIC) [15].

The hybrid of co-located mMIMO and NOMA offers a great potential to support low latency massive connectivity requirements of the next-generation wireless networks while further improving the spectral efficiency of NOMA-based systems [17]-[19]. Thus, integrating NOMA with CF mMIMO may reap further gains and is therefore a critically important research topic.

\section{B. Problem Statement and Contributions}

Hybrid CF massive MIMO-NOMA offers tremendous potential to improve the spectral efficiency. To achieve this goal, two key factors are essential. First, precoding of superpositioncoded signals to multiple user-clusters is necessary. Second, the user multiplexing in each cluster must be on the basis of their channel power gain differentials. However, so far such 
hybrid designs have been investigated by only few papers [20]-[22]. Thus, the main objective of this paper is to add to this literature and further investigate these systems.

In particular, for CF massive MIMO-NOMA systems, we investigate three linear precoding schemes, with the same front-hauling overhead. These are maximum ratio transmission (MRT), full-pilot zero-forcing (fpZF) and modified regularized ZF (mRZF). Of these three, while its performance in mMIMO systems has been investigated [23]-[25], mRZF has not been studied for $\mathrm{CF}$ massive MIMO before. The advantages of mRZF include its ability to balance the interference suppression and desired signal power and also having additional parameters to be optimized.

Normal ZF and RZF precoders require exchanging instantaneous channel state information (CSI) among the APs - the main benefit of mRZF and fpZF is the elimination of this exchange and that each AP computes its precoder with only its local CSI. This is a big advantage as mRZF and fpZF have the same front-hauling overhead as MRT [26], [27]. Unlike MRT which maximizes the signal gain at the intended cluster and ignores the inter-cluster interference, fpZF sacrifices some of the array gain to cancel the inter-cluster interference [26], [27]. On the other hand, mRZF balances the inter-cluster interference mitigation and intra-cluster power enhancement. Note that the optimal precoding is nonlinear, so these linear precoders are sub-optimal. But they offer high performance with affordable computational complexity, making them ideal for practical large MIMO systems [23].

The main contributions of this paper on CF massive MIMONOMA systems can be summarized as follows:

1) We derive the closed-form downlink sum rate when the APs employ MRT or fpZF precoders, considering the effects of intra-cluster pilot contamination, and intercluster interference. The users rely on the statistics for their effective channels for decoding, and the SIC process is imperfect.

2) We also analyze the performance when the APs employ mRZF precoding. Since the closed-form analysis of it with finite system parameters is difficult (if not impossible), we analyze the achievable rate when the number of clusters $(N)$ and the number of antennas at each AP $(L)$ grow infinitely large while $\frac{L}{N}$ is a finite ratio.

3) We show that NOMA allows a significant number of users to be supported simultaneously at the same coherence interval compared to its counterpart OMA. For instance, with $K$ users in each cluster, NOMA based CF massive MIMO can support $K$ times the users that of OMA. Moreover, for a large number of users, NOMA outperforms OMA; however, for low number of users, the sum rate of NOMA is lower than that of OMA due to the effects of intra-cluster pilot contamination and imperfect SIC. It is further shown that given perfect SIC, mRZF and fpZF significantly outperform MRT. We also show that either mRZF or fpZF outperforms OMA systems with MRT.

4) Numerical results are also presented to support our findings.

\section{Previous Contributions on CF massive MIMO-NOMA}

As mentioned before, the investigation of $\mathrm{CF}$ massive MIMO-NOMA systems has been sparse, except for [20]-[22]. In fact [20] is the first paper to study the design of such systems. It considers single-antenna APs that use conjugate beamforming precoders. It thus analyzes degradations due to estimated/imperfect CSI at APs, SIC, and statistical CSI at users. On the other hand, [21] generalizes to multipleantenna APs and derives minimum mean square error (MMSE) uplink and downlink channel estimates, and for both these cases, the achievable rate is derived. Reference [21] also considers spatial correlation among the multiple antennas at each AP and intra-cluster pilot contamination and quantifies the adverse impact of intra-cluster pilot contamination and error propagation due to imperfect SIC at the user nodes. The only other work [22] deals with the max-min fairness based bandwidth efficiency problem. It develops an optimal algorithm and mode switching between NOMA and OMA for maximum bandwidth efficiency.

The above paragraph clarifies the differences between this paper and [20]-[22]. The focus of this paper is to comparatively evaluate the three types of practical precoders. We believe that this paper is the first to do that in the context of CF massive MIMO-NOMA.

\section{General works on MIMO NOMA}

Since the NOMA literature is vast, we mention only few works here. NOMA outperforms OMA from user and system throughput perspectives [28]. In [29], multi-user power allocation, user scheduling, and error propagation in SIC for NOMA are investigated. MIMO-NOMA outperforms conventional MIMO-OMA systems [30], [31]. MIMO-NOMA outperforms MIMO-OMA in terms of both sum channel capacity and ergodic sum capacity [30]. Furthermore, the user outage probability in a MIMO-NOMA cluster is studied in [31]. Beamforming, user clustering, and power allocation for MIMO-NOMA are further investigated in [32].

Hybrid massive MIMO-NOMA, which outperforms standalone mMIMO and NOMA schemes, has been studied in [19], [33]. The outage probability with perfect user ordering and limited feedback is investigated in [34]. In [17], a user pairing and pair scheduling algorithm is proposed to enhance the spectral efficiency of massive MIMO-NOMA systems. Moreover, in [18], user clustering and pilot assignment schemes for multi-cell massive MIMO-NOMA are investigated by employing the characteristics of correlated fading channels.

\section{E. Structure and Notations}

This paper is organized as follows. The system model is introduced in Section II. In Section III the achievable sum rate with linear precoding techniques is derived. In Section IV, the analytical results are confirmed through simulation examples. Section V] concludes the paper.

Notation: Lower-case bold and upper-case bold denote vectors and matrices, respectively. $\mathbf{I}_{n}$ represents the $n \times n$ 


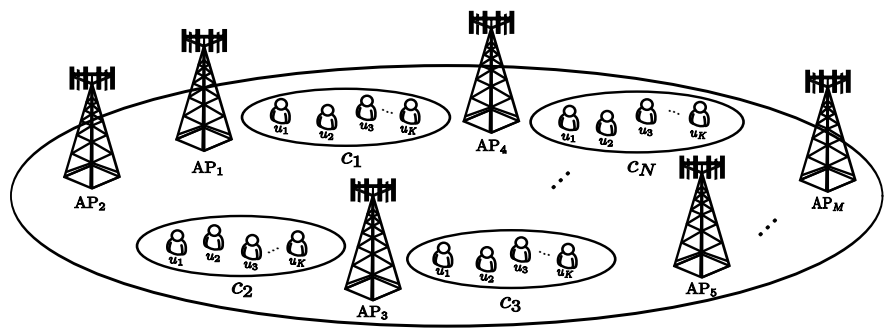

(a)

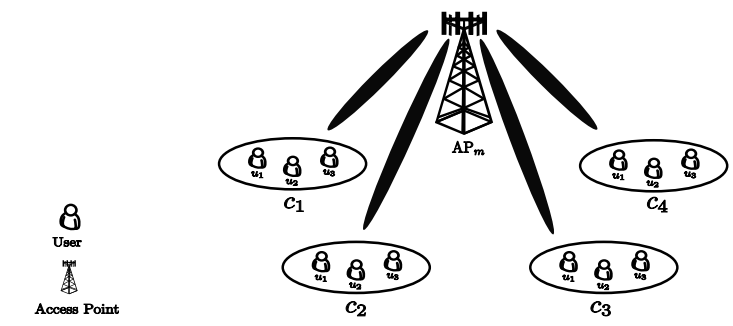

(b)

Fig. 1. (a) System model of cell-free massive MIMO-NOMA (b) An AP transmits to four clusters $\left\{c_{1}, \ldots, c_{4}\right\}$, each with three users.

identity matrix. $\mathbf{A}^{\mathrm{T}}, \mathbf{A}^{\mathrm{H}}, \operatorname{tr}[\mathbf{A}]$, and $[\mathbf{A}]_{(m, n)}$ denote transpose, Hermitian transpose, trace, and the $(m, n)$ th element of matrix $\mathbf{A}$, respectively. $\mathbb{E}\{\cdot\}$ denotes the statistical expectation. Finally, $\mathcal{C N}(\boldsymbol{\mu}, \mathbf{R})$ is a complex Gaussian vector with mean $\boldsymbol{\mu}$ and co-variance matrix $\mathbf{R}$.

\section{System Model AND PRELIMINARIES}

Here, we describe the system model, channel model, and transmission model in detail.

\section{A. System and Channel Models}

Consider the downlink transmission of CF massive MIMONOMA. This system has $M$ APs and $K N$ single-antenna users, which are grouped into $N$ clusters with $K(K \geq 2)$ users per cluster and NOMA is applied among the users in the same cluster. Each AP is equipped with $L$ antennas (Fig. 1. a). The total $M$ APs serve $K N$ users in the same timefrequency resource block, where $K N \ll M L$. All the APs are connected to a CPU via an error-free fronthaul network to achieve coherent processing [8]. This network carries only the payload data, large-scale parameters and power control coefficients that change slowly. Each AP computes its precoder based on the estimates of the channel states between itself and the users. Importantly, it does not need the knowledge of the channel states between the users and other APs.

The downlink channel between the $m$ th AP $(m=1, \ldots, M)$ and the $k$ th user $(k=1, \ldots, K)$ in the $n$th cluster $(n=$ $1, \ldots, N)$ is the complex Gaussian random vector

$$
\mathbf{h}_{m n k} \sim \mathcal{C N}\left(\mathbf{0}, \beta_{m n k} \mathbf{I}_{L}\right),
$$

where $\left\{\beta_{m n k}\right\}$ are the set of the large-scale fading coefficients. Each AP is assumed to know its own set of large-scale coefficients [8]. This assumption is justifiable because they are quasi-static and hence only need to be estimated once about every 40 coherence time intervals [35]. This model (1) also presumes that small-scale fading is Rayleigh distributed.

\section{B. Uplink Pilot Transmission}

The users transmit pilot sequences in the uplink, and the APs use them to estimate the downlink channels. In order to minimize the channel estimation overhead in NOMA, the same pilot sequence of length $\tau$ samples, is shared among the users within each cluster, but different clusters are assigned mutually orthogonal pilots ${ }^{1}[20]$. Then, the pilot sequence for the $k$ th user in the $n$th cluster is $\sqrt{\tau} \phi_{n} \in \mathcal{C}^{\tau \times 1}$ satisfying $\left\|\phi_{n}\right\|^{2}=1$ where $N \leq \tau$. Accordingly, the pilot sequences allocated to the $N$ clusters are mutually orthogonal implying that $\phi_{n}^{\mathrm{H}} \phi_{j}=0(n \neq j)$. The received pilot signal at the $m$ th AP $\left(\mathbf{Y}_{m}^{p} \in \mathcal{C}^{L \times \tau}\right)$ can then be expressed as

$$
\mathbf{Y}_{m}^{p}=\sqrt{\tau p_{p}} \sum_{n=1}^{N} \sum_{k=1}^{K} \mathbf{h}_{m n k} \boldsymbol{\phi}_{n}^{\mathrm{H}}+\mathbf{N}_{m}, \quad \forall m
$$

where $p_{p}$ is the pilot transmit power and $\mathbf{N}_{m} \in \mathcal{C}^{L \times \tau}$ is a Gaussian noise matrix with i.i.d $\mathcal{C N}(0,1)$ elements. Then, the $m$ th AP estimates $\mathbf{h}_{m n k}$ using minimum mean square error (MMSE) estimation. To do so, the received pilot signal at the $m$ th AP (2) is projected onto $\phi_{n}$ which yields

$$
\tilde{\mathbf{y}}_{m n}^{p}=\sqrt{\tau p_{p}} \sum_{k=1}^{K} \mathbf{h}_{m n k}+\tilde{\mathbf{n}}_{m n}
$$

where $\tilde{\mathbf{n}}_{m n}=\mathbf{N}_{m} \boldsymbol{\phi}_{n} \sim \mathcal{C N}\left(\mathbf{0}, \mathbf{I}_{L}\right)$.

By using (3), the MMSE estimate of $\mathbf{h}_{m n k}$ can be expressed as $\hat{\mathbf{h}}_{m n k}=c_{m n k} \tilde{\mathbf{y}}_{m n}^{p}$, where $c_{m n k}$ is given by

$$
c_{m n k}=\frac{\sqrt{\tau p_{p}} \beta_{m n k}}{1+\tau p_{p} \sum_{i=1}^{K} \beta_{m n i}} .
$$

Since $\tilde{\mathbf{y}}_{m n}^{p}$ is Gaussian distributed, $\hat{\mathbf{h}}_{m n k}$ can be written as,

$$
\hat{\mathbf{h}}_{m n k}=\sqrt{\theta_{m n k}} \nu_{m n},
$$

where $\nu_{m n} \sim \mathcal{C N}\left(\mathbf{0}, \mathbf{I}_{L}\right)$ and $\theta_{m n k}$ is equal to

$$
\theta_{m n k}=\frac{1}{L} \mathbb{E}\left\{\left\|\hat{\mathbf{h}}_{m n k}\right\|^{2}\right\}=\frac{\tau p_{p} \beta_{m n k}^{2}}{1+\tau p_{p} \sum_{i=1}^{K} \beta_{m n k}} .
$$

The channel estimation error can then be defined as $\boldsymbol{\epsilon}_{m n k}=$ $\mathbf{h}_{m n k}-\hat{\mathbf{h}}_{m n k}$ where $\boldsymbol{\epsilon}_{m n k} \sim \mathcal{C N}\left(\mathbf{0},\left(\beta_{m n k}-\theta_{m n k}\right) \mathbf{I}_{L}\right)$. Since the users in the same cluster use the same pilot sequence, their channels are parallel as shown in (5). Mathematically, this can be written as

$$
\hat{\mathbf{h}}_{m n k}=\frac{\beta_{m n k}}{\beta_{m n i}} \hat{\mathbf{h}}_{m n i},
$$

where $\beta_{m n k}$ is already given by (1).

\section{Downlink Data Transmission Model}

The superposition coded data signal for the $K$ users in the $n$th cluster is expressed as

$$
s_{n}=\sum_{k=1}^{K} \sqrt{p_{n k}} s_{n k}, \quad \forall n
$$

${ }^{1}$ It is different from OMA where all the users in all the clusters are assigned with different mutually orthogonal pilots. 
In (8), $s_{n k}$ and $p_{n k}$ denote the data signal and the transmitted power allocated to the $k$ th user in the $n$th cluster. Also, $p_{n k}=p_{t} \lambda_{n k}$ where $p_{t}$ is the total transmit power of each $\mathrm{AP}$ and $\left\{\lambda_{n k}\right\}$ are the set of power coefficients that satisfies $\sum_{n=1}^{N} \sum_{k=1}^{K} \lambda_{n k}=1$. Furthermore, the different data signals are mutually uncorrelated:

$$
\mathbb{E}\left\{s_{n k} s_{m i}^{*}\right\}=\left\{\begin{array}{ll}
1, & m=n \quad \& \quad k=i \\
0, & \text { else }
\end{array},\right.
$$

where $m, n \in\{1,2, \ldots, N\}$ and $k, i \in\{1,2, \ldots, K\}$. Therefore, $\mathbb{E}\left\{\left|s_{n}\right|^{2}\right\}=\sum_{k=1}^{K} p_{n k}=p_{t} \lambda_{n}$, where $\lambda_{n}=\sum_{k=1}^{K} \lambda_{n k}$ accounts for the power allocation coefficient for the $n$th cluster.

The $m$ th AP $(m=1, \ldots, M)$ transmits the signal

$$
\mathbf{x}_{m}=\sum_{n=1}^{N} \mathbf{w}_{m n} s_{n}
$$

where $\mathbf{w}_{m n} \in \mathcal{C}^{L}$ represents the spatial directivity of the signals sent to the users in the $n$th cluster. Note that each AP precodes the transmitted signals for all the users in the same cluster with the same beamforming vector $\mathbf{w}_{m n}$, i.e., each AP has $N$ precoding vectors (Fig. 11 b).

Since the $K N$ users are served simultaneously by $M$ APs, the received signal at the $k$ th user in the $n$th cluster can be expressed as

$$
\begin{aligned}
y_{n k} & =\underbrace{\sum_{m=1}^{M} \sqrt{p_{n k}} \mathbf{h}_{m n k}^{\mathrm{H}} \mathbf{w}_{m n} s_{n k}}_{\text {desired signal }}+\underbrace{\sum_{m=1}^{M} \mathbf{h}_{m n k}^{\mathrm{H}} \mathbf{w}_{m n} \sum_{\substack{i=1 \\
i \neq k}}^{K} \sqrt{p_{n i}} s_{n i}}_{\text {intra-cluster interference before SIC }} \\
& +\underbrace{\sum_{\substack{n^{\prime}=1 \\
n^{\prime} \neq n}}^{M} \mathbf{h}_{m n k}^{\mathrm{H}} \sum_{m=1}^{N} \mathbf{w}_{m n^{\prime}} s_{n^{\prime}}}_{\text {inter-cluster interference }}+n_{n k} .
\end{aligned}
$$

In (11), $n_{n k} \sim \mathcal{C N}(0,1)$ while the first, second, and the third terms are desired signal, intra-cluster interference before SIC and inter-cluster interference, respectively.

In TDD CF massive MIMO systems, with sufficiently large number antennas at each AP (e.g., 5 - 10 antennas per AP [36]), the instantaneous channel coefficients can be well approximated by their corresponding expected values. This phenomenon is referred to as "channel hardening" which substantially reduces the effective channel gain fluctuations; thereby rendering the use of downlink pilot training unnecessary [8], [36]. To apply power domain NOMA, we thus assume that the users in the $n$th cluster are ordered based on the mean of the effective channel gains as follows [20], [21]:

$$
\Gamma_{n 1} \geq \Gamma_{n 2} \geq \cdots \geq \Gamma_{n K}
$$

where $\Gamma_{n k}=\mathbb{E}\left\{\left|\sum_{m=1}^{M} \hat{\mathbf{h}}_{m n k}^{\mathrm{H}} \mathbf{w}_{m n}\right|^{2}\right\}$.

The user ordering can be done by a central entity (e.g., the CPU) that collects all information of the mean of effective channel strengths and then feeds back the result of user ordering and power allocation to the APs; higher powers are allocated to the users with lower channel strength; i.e., $p_{n 1} \leq p_{n 2} \leq \cdots \leq p_{n K}$. Hence, the $k$ th user applies
SIC to decode its own signal. More precisely, it decodes the signals of the users with higher powers and treats the others as interference. In particular, the $k$ th user decodes the signal intended for the $i$ th user $(\forall i \geq k)$ and treats the signals of the other users $(\forall i<k)$ as interference.

Using the statistical CSI knowledge of the effective channels at users, i.e., $\mathbb{E}\left\{\mathbf{h}_{m n k}^{\mathrm{H}} \mathbf{w}_{m n}\right\}(\forall m, n, k),(11)$ can be rewritten as follows:

$$
\begin{aligned}
y_{n k} & =\underbrace{\sum_{m=1}^{M} \sqrt{p_{n k}} \mathbb{E}\left\{\mathbf{h}_{m n k}^{\mathrm{H}} \mathbf{w}_{m n}\right\} s_{n k}}_{T_{0}: \text { desired signal }} \\
& +\underbrace{\sum_{m=1}^{M} \sqrt{p_{n k}}\left(\mathbf{h}_{m n k}^{\mathrm{H}} \mathbf{w}_{m n}-\mathbb{E}\left\{\mathbf{h}_{m n k}^{\mathrm{H}} \mathbf{w}_{m n}\right\}\right) s_{n k}}_{T_{1}: \text { beamforming gain uncertainty }} \\
& +\underbrace{\sum_{m=1}^{M} \sum_{i=1}^{k-1} \sqrt{p_{n i}} \mathbf{h}_{m n k}^{\mathrm{H}} \mathbf{w}_{m n} s_{n i}} \\
& +\underbrace{\sum_{m=1}^{M} \sum_{i=k+1}^{K}{\sqrt{p_{n i}}}_{T_{4}: \text { Inter-cluster interference }}\left(\mathbf{h}_{m n k}^{\mathrm{H}} \mathbf{w}_{m n} s_{n i}-\mathbb{E}\left\{\mathbf{h}_{m n k}^{\mathrm{H}} \mathbf{w}_{m n}\right\}\right.}_{T_{2}: \text { intra-cluster interference after SIC }} \hat{s}_{n i}) \\
& +\sum_{T_{3}: \text { residual interference due to imperfect SIC }}^{\sum_{m=1}^{M} \mathbf{h}_{m n k}^{\mathrm{H}} \sum_{\substack{n^{\prime}=1 \\
n^{\prime} \neq n}}^{N} \mathbf{w}_{m n^{\prime}} s_{n^{\prime}}+n_{n k}},
\end{aligned}
$$

where the first term $T_{0}$ is the desired signal and the second term $T_{1}$ is the beamforming gain uncertainty. The third term $T_{2}$ is intra-cluster interference caused by the signals of the users which are considered as the interference at the $k$ th user. Note that because the users know statistical CSI only, intracluster pilot contamination and channel estimation error, SIC may not be perfect. The forth term $T_{3}$ thus represents the error propagation due to the imperfect SIC where $\hat{s}_{n i}$ is the estimate of $s_{n i}$. Here, we adopt the linear MMSE estimation [37] where $s_{n i}$ and its estimate $\hat{s}_{n i}$ can be assumed as jointly Gaussian distributed with a normalized correlation coefficient. The relationship between $s_{n i}$ and $\hat{s}_{n i}$ can then be written as

$$
s_{n i}=\rho_{n i} \hat{s}_{n i}+e_{n i},
$$

where $\hat{s}_{n i} \sim \mathcal{C N}(0,1), e_{n i} \sim \mathcal{C N}\left(0, \sigma_{e_{n i}}^{2} /\left[1+\sigma_{e_{n i}}^{2}\right]\right)$ is the estimation error, statistically independent of $\hat{s}_{n i}$, and $\rho_{n i}=$ $1 / \sqrt{1+\sigma_{e_{n i}}^{2}}$. The correlation coefficient $0 \leq \rho \leq 1$ reflects the quality of the estimation and quantify the severity of the SIC imperfection. The value of $\rho$ is determined by channel related issues (fading and shadowing) and other factors [38]; the greater its value, the greater the correlation between $\hat{s}_{n i}$ and $s_{n i}$ and better the SIC performance [20].

\section{Downlink Achievable Rate}

Here, we analyze the downlink sum rates achievable with MRT, fpZF, and mRZF. To this end, we first derive the effective signal-to-interference-plus-noise ratio (SINR) at each user. It then leads to the total downlink sum rate. 
According to (13), since data signals intended to different users are mutually uncorrelated and the white additive noise is independent from the data symbols and the channel coefficients, it is easy to check that $T_{i}, \forall i$ and $n_{n k}$ are mutually uncorrelated. Therefore, by considering the first term in (13) as the desired signal and the remaining terms as an effective noise, invoking the argument in [39], the SINR at the $k$ th user in the $n$th cluster $\gamma_{n k}$ can be derived as (15), shown at the top of the next page, where $\eta_{n k}$ is defined as $\eta_{n k} \triangleq \sum_{m=1}^{M} \mathbf{h}_{m n k}^{\mathrm{H}} \mathbf{w}_{m n}$. The achievable rate for the $k$ th user in the $n$th cluster can then be computed as

$$
\mathcal{R}_{n k}=\zeta \log _{2}\left(1+\gamma_{n k}\right),
$$

where $\zeta=\left(\tau_{c}-\tau\right) / \tau_{c}$ is the pre-log factor and $\tau_{c}$ is the coherence interval.

The achievable rate $\mathcal{R}_{n k}$ in 16 depends on the precoding process at the APs. In order to design the precoding matrices at the APs, we first define $\overline{\mathbf{H}}_{m} \triangleq \mathbf{Y}_{m}^{p} \Phi$ where $\boldsymbol{\Phi}=\left[\boldsymbol{\phi}_{1}, \phi_{2}, \ldots, \boldsymbol{\phi}_{N}\right] \in \mathcal{C}^{\tau \times N}$ and $\mathbf{Y}_{m}^{p} \in \mathcal{C}^{L \times \tau}$ is given by (2). $\overline{\mathbf{H}}_{m}$ can be then written as

$\overline{\mathbf{H}}_{m}=\left[\sqrt{\tau p_{p}} \sum_{k=1}^{K} \mathbf{h}_{m 1 k}+\tilde{\mathbf{n}}_{m}, \ldots, \sqrt{\tau p_{p}} \sum_{k=1}^{K} \mathbf{h}_{m N k}+\tilde{\mathbf{n}}_{m}\right]$.

Therefore, $\overline{\mathbf{H}}_{m}$ has independent columns and $\overline{\mathbf{h}}_{m n} \sim$ $\mathcal{C N}\left(\mathbf{0},\left(1+\tau p_{p} \sum_{k=1}^{k} \beta_{m n k}\right) \mathbf{I}_{L}\right)$ is the $n$th column of $\overline{\mathbf{H}}_{m}$ i.e., $\overline{\mathbf{h}}_{m n}=\overline{\mathbf{H}}_{m} \mathbf{e}_{n}$ where $\mathbf{e}_{n}$ denotes the $n$th column of $\mathbf{I}_{N}$. Then, the channel estimate can be written as

$$
\hat{\mathbf{h}}_{m n k}=c_{m n k} \overline{\mathbf{h}}_{m n} .
$$

We next assume that the APs use either MRT, fpZF or mRZF to precode data signals. For each precoder, we then derive the closed-form achievable sum rate.

\section{A. MRT beamforming}

With MRT, the $m$ th AP computes the following precoding vector for the $k$ th user in the $n$th cluster:

$$
\mathbf{w}_{m n}=\frac{\overline{\mathbf{h}}_{m n}}{\sqrt{\mathbb{E}\left\{\left\|\overline{\mathbf{h}}_{m n}\right\|^{2}\right\}}} .
$$

This precoder (19) is used for all the users in the $n$th cluster.

Theorem 1. In the CF massive MIMO-NOMA system with MRT precoding, $\gamma_{n k}$ in (15), for finite values of $M, L, N$ and $K$, is given by (20), as shown at the top of the next page.

Proof. See Appendix A for derivations.

Since 20 connects several factors together, it can be used to provide several remarks and guidelines relevant for practical NOMA-based CF mMIMO. We briefly describe them next.

Remark 1. With MRT precoding, the signal power increases as the number of antennas at each AP, L, increases, thanks to the array gain. On the other hand, the interference due to the pilot contamination and imperfect SIC (the first term in the denominator) proportionally increases as $L$ increases.
Remark 2. Beamforming gain uncertainty and inter-cluster interference (the second term in the denominator), are not affected by $L$.

Remark 3. As opposed to the number of clusters $N$, which is restricted by the maximum orthogonal pilot sequence length $(N \leq \tau)$, the number of users $K$ within each cluster can be increased greatly. However, the downside is the increase of pilot contamination and imperfect SIC.

\section{B. Full-pilot $Z F$ beamforming}

In this case, the precoding vector at the $m$ th AP for the users in the $n$th cluster can be formulated as

$$
\mathbf{w}_{m n}=\frac{\overline{\mathbf{H}}_{m}\left(\overline{\mathbf{H}}_{m}^{\mathrm{H}} \overline{\mathbf{H}}_{m}\right)^{-1} \mathbf{e}_{n}}{\sqrt{\mathbb{E}\left\{\left\|\overline{\mathbf{H}}_{m}\left(\overline{\mathbf{H}}_{m}^{\mathrm{H}} \overline{\mathbf{H}}_{m}\right)^{-1} \mathbf{e}_{n}\right\|^{2}\right\}}},
$$

where each AP has $N$ precoding vectors, one per cluster (pilot).

In order to find the effective SINR $\gamma_{n k}$ given in (15), we first need to derive the value of $\eta_{n k}, \forall n$. To obtain $\eta_{n k}, \forall n$, we need to compute $\hat{\mathbf{h}}_{m n k}^{\mathrm{H}} \mathbf{w}_{m n^{\prime}}$. To this end, we first obtain the normalization term in (21). By employing Lemma 2.10 of [40] and using the properties of $N \times N$ central complex Wishart matrix with $L(L \geq N+1)$ degrees of freedom, we obtain

$$
\begin{aligned}
& \mathbb{E}\left\{\left\|\overline{\mathbf{H}}_{m}\left(\overline{\mathbf{H}}_{m}^{\mathrm{H}} \overline{\mathbf{H}}_{m}\right)^{-1} \mathbf{e}_{n}\right\|^{2}\right\}=\mathbb{E}\left\{\left(\overline{\mathbf{H}}_{m}^{\mathrm{H}} \overline{\mathbf{H}}_{m}\right)_{n, n}^{-1}\right\} \\
& =\frac{1}{(L-N)\left(1+\tau p_{p} \sum_{k=1}^{K} \beta_{m n k}\right)} .
\end{aligned}
$$

Finally, by employing (18), 21) and (22), we have

$$
\begin{aligned}
\hat{\mathbf{h}}_{m n k}^{\mathrm{H}} \mathbf{w}_{m n^{\prime}} & =c_{m n k} \mathbf{e}_{n}^{\mathrm{H}} \mathbf{e}_{n^{\prime}} \sqrt{(L-N)\left(1+\tau p_{p} \sum_{k=1}^{K} \beta_{m n k}\right)} \\
& = \begin{cases}\sqrt{\theta_{m n k}(L-N)}, & n=n^{\prime} \\
0, & n \neq n^{\prime}\end{cases}
\end{aligned}
$$

From 23, we find that fpZF precoder suppresses the intercluster interference by only utilizing local CSI rather than CSI shared among the APs, a big advantage. Moreover, fpZF has the same front-hauling overhead as MRT.

Theorem 2. In the CF massive MIMO-NOMA system with fpZF precoder, $\gamma_{n k}$ in (15), for any finite $M, L, N$ and $K$, is given by (24) as shown at the top of the next page.

Proof. See Appendix B for the derivations.

Remark 4. Similar to MRT, by employing fpZF precoding, the signal power increases as the number of antennas at each AP L increases, thanks to the array gain (which is $L-N)$. On the other hand, the interference due to the pilot contamination and imperfect SIC (the first term in the denominator) proportionally increases as $L$ increases.

Remark 5. Unlike MRT which only aims to maximize the signal-to-noise ratio (SNR) and ignores the inter-cluster interference, fpZF aims to suppress the inter-cluster interference by sacrificing array gain. 


$$
\gamma_{n k}=\frac{p_{n k}\left|\mathbb{E}\left\{\eta_{n k}\right\}\right|^{2}}{p_{n k} \mathbb{E}\left\{\left|\left(\eta_{n k}-\mathbb{E}\left\{\eta_{n k}\right\}\right)\right|^{2}\right\}+\sum_{i=1}^{k-1} p_{n i} \mathbb{E}\left\{\left|\eta_{n k}\right|^{2}\right\}+\sum_{i=k+1}^{K} p_{n i} \mathbb{E}\left\{\left|\eta_{n k} s_{n i}-\mathbb{E}\left\{\eta_{n k}\right\} \hat{s}_{n i}\right|^{2}\right\}+\sum_{\substack{n^{\prime}=1 \\ n^{\prime} \neq n}}^{N} p_{n^{\prime}} \mathbb{E}\left\{\left|\eta_{n^{\prime} k}\right|^{2}\right\}+1} .
$$

$$
\gamma_{n k}^{\mathrm{MRT}}=\frac{L p_{n k}\left(\sum_{m=1}^{M} \sqrt{\theta_{m n k}}\right)^{2}}{L\left(\sum_{m=1}^{M} \sqrt{\theta_{m n k}}\right)^{2}\left(\sum_{i=1}^{k-1} p_{n i}+\sum_{i=k+1}^{K} p_{n i}\left(2-2 \rho_{n i}\right)\right)+\left(\sum_{n^{\prime}=1}^{N} p_{n^{\prime}}\right) \sum_{m=1}^{M} \beta_{m n k}+1}
$$

$$
\gamma_{n k}^{\mathrm{fpZF}}=\frac{(L-N) p_{n k}\left(\sum_{m=1}^{M} \sqrt{\theta_{m n k}}\right)^{2}}{(L-N)\left(\sum_{m=1}^{M} \sqrt{\theta_{m n k}}\right)^{2}\left(\sum_{i=1}^{k-1} p_{n i}+\sum_{i=k+1}^{K} p_{n i}\left(2-2 \rho_{n i}\right)\right)+\left(\sum_{n^{\prime}=1}^{N} p_{n^{\prime}}\right) \sum_{m=1}^{M}\left(\beta_{m n k}-\theta_{m n k}\right)+1} .
$$

Remark 6. For a fixed number of clusters $N$, if the number of antennas at each AP tends to infinity $(L \rightarrow \infty)$, the SINR for both the MRT and fpZF precoding (20) and 24) can be approximated as

$$
\lim _{L \rightarrow \infty} \gamma_{n k}=\frac{p_{n k}\left(\sum_{m=1}^{M} \sqrt{\theta_{m n k}}\right)^{2}}{\left(\sum_{m=1}^{M} \sqrt{\theta_{m n k}}\right)^{2}\left(\sum_{i=1}^{k-1} p_{n i}+\sum_{i=k+1}^{K} p_{n i}\left(2-2 \rho_{n i}\right)\right)} .
$$

which shows that, the gain of adding more antennas at each $A P$ disappears. Besides, for fixed values of $L$ and $N$, by increasing the number of users at each cluster, pilot contamination and SIC become the dominant interferences. Therefore, the performance of the CF massive MIMO-NOMA system is limited by pilot contamination and imperfect SIC.

In contrast, in OMA, where each user is assigned with an orthogonal pilot, SINR $\gamma_{n k}$ for MRT and fpZF is given as

$$
\gamma_{n k, \mathrm{OMA}}^{\mathrm{MRT}}=\frac{L p_{n k}\left(\sum_{m=1}^{M} \sqrt{\theta_{m n k}}\right)^{2}}{\left(\sum_{n^{\prime}=1}^{N} \sum_{k^{\prime}=1}^{K} p_{n^{\prime} k^{\prime}}\right) \sum_{m=1}^{M} \beta_{m n k}+1}
$$

and

$$
\gamma_{n k, \text { OMA }}^{\mathrm{fpZF}}=\frac{(L-K N) p_{n k}\left(\sum_{m=1}^{M} \sqrt{\theta_{m n k}}\right)^{2}}{\left(\sum_{n^{\prime}=1}^{N} \sum_{k^{\prime}=1}^{K} p_{n^{\prime} k^{\prime}}\right) \sum_{m=1}^{M}\left(\beta_{m n k}-\theta_{m n k}\right)+1} .
$$

Therefore, deploying more antennas at each AP is always beneficial for OMA.

\section{Modified RZF beamforming}

The mRZF precoder tries to balance inter-cluster interference mitigation and intra-cluster power enhancement. Since closed-form analysis of it is difficult (if not impossible), we analyze its achievable rate in the asymptotic regime, where the number of clusters $N$ and the number of antennas at each AP $L$ grows infinitely large while keeping a finite ratio; i.e., $1 \leq \lim _{L, N \rightarrow \infty} \frac{L}{N} \leq \propto^{2}$. This asymptotic expression can nevertheless be used with finite values of $L$ and $N$ [23], [24].

The precoding vector of mRZF at the $m$ th AP for the users in the $n$th cluster can be expressed as

$$
\mathbf{w}_{m n}=\frac{\left(\overline{\mathbf{H}}_{m} \overline{\mathbf{H}}_{m}^{\mathrm{H}}+L \alpha \mathbf{I}_{L}\right)^{-1} \overline{\mathbf{h}}_{m n}}{\sqrt{\mathbb{E}\left\{\left\|\left(\overline{\mathbf{H}}_{m} \overline{\mathbf{H}}_{m}^{\mathrm{H}}+L \alpha \mathbf{I}_{L}\right)^{-1} \overline{\mathbf{h}}_{m n}\right\|^{2}\right\}}},
$$

where $\overline{\mathbf{H}}_{m}$ is given in (17) and $\alpha>0$ is the regularization parameter that can be optimized [41]. Finding optimal value for $\alpha$, however, is outside the scope of this paper and is left for future work. Here, we assume that $\alpha$ is scaled with $L$ to ensure that it converges to a constant value as $L$ and $N$ tend to infinity.

It can be seen from (28), in our design the precoder of each AP only utilizes local CSI rather than global CSI knowledge shared between the APs. Hence, mRZF has the same fronthauling overhead as that of MRT and fpZF.

Theorem 3. In the CF massive MIMO-NOMA system with $m R Z F$ precoder, $\gamma_{n k}$ in 150 , when $L$ and $N$ grow large such that $1 \leq \lim _{L, N \rightarrow \infty} \frac{L}{N} \leq \infty$, is given by 29] as shown at the top of the next page, where $\boldsymbol{\Theta}_{m n}=\left(1+\tau p_{p} \sum_{k=1}^{k} \beta_{m n k}\right) \mathbf{I}_{L}$, $a_{m n k}=\sqrt{\frac{\beta_{m n k}-\theta_{m n k}}{1+\tau p_{p} \sum_{k=1}^{K} \beta_{m n k}}}$ and $e_{m n}^{o}=e_{m n}$ in which

$$
e_{m n}=\frac{1}{L} \operatorname{tr}\left[\boldsymbol{\Theta}_{m n} \mathbf{T}_{m}\right]
$$

\footnotetext{
${ }^{2}$ This assumption implies that the coherence time of the channel $\tau_{c}$ scales linearly with $N$. However, as it will be shown in Section IV, our analysis provides a tight approximation for the achievable rate even for small values of $N$ or equivalently $\tau_{c}$.
} 


$$
\gamma_{n k}^{\mathrm{mRZF}}=\frac{p_{n k}\left(\sum_{m=1}^{M} \frac{1}{\sqrt{\psi_{m n}^{o}}} \frac{c_{m n k} e_{m n}^{o}}{1+e_{m n}^{o}}\right)^{2}}{\left(\sum_{m=1}^{M} \frac{1}{\sqrt{\psi_{m n}^{o}}} \frac{c_{m n k} e_{m n}^{o}}{1+e_{m n}^{o}}\right)^{2}\left(\sum_{i=1}^{k-1} p_{n i}+\sum_{i=k+1}^{K} p_{n i}\left(2-2 \rho_{n i}\right)\right)+\sum_{m=1}^{M} \Upsilon_{m n}\left(\frac{c_{m n k}^{2}}{\left(1+e_{m n}^{o}\right)^{2}}+a_{m n k}^{2}\right)+1} .
$$

$$
\begin{gathered}
\mathbf{T}_{m}=\left(\frac{1}{L} \sum_{j=1}^{N} \frac{\Theta_{m j}}{1+e_{m j}}+\alpha \mathbf{I}_{L}\right)^{-1} \\
\psi_{m n}^{o}=\frac{1}{L} \frac{e_{m n}^{\prime}}{\left(1+e_{m n}\right)^{2}} \\
\Upsilon_{m n}=\frac{1}{L} \sum_{\substack{n^{\prime}=1 \\
n^{\prime} \neq n}}^{N} \frac{p_{n^{\prime}} e_{n^{\prime}, m n}^{\prime}}{\psi_{m n^{\prime}}^{o}\left(1+e_{m n^{\prime}}\right)^{2}}
\end{gathered}
$$

in which $\mathbf{e}_{m}^{\prime}=\left[e_{m 1}^{\prime}, \ldots, e_{m N}^{\prime}\right]^{\mathrm{T}}$ and $\mathbf{e}_{m n}^{\prime}=$ $\left[e_{1, m n}^{\prime}, \ldots, e_{N, m n}^{\prime}\right]^{\mathrm{T}}$ are given by

$$
\begin{aligned}
\mathbf{e}_{m}^{\prime} & =\left(\mathbf{I}_{N}-\mathbf{J}_{m}\right)^{-1} \mathbf{v}_{m}, \\
\mathbf{e}_{m n}^{\prime} & =\left(\mathbf{I}_{N}-\mathbf{J}_{m}\right)^{-1} \mathbf{v}_{m n},
\end{aligned}
$$

and $\mathbf{J}_{m}, \mathbf{v}_{m}$ and $\mathbf{v}_{m n}$ are derived as follows,

$\left[\mathbf{J}_{m}\right]_{i j}=\frac{\frac{1}{L} \operatorname{tr}\left[\boldsymbol{\Theta}_{m i} \mathbf{T}_{m} \boldsymbol{\Theta}_{m j} \mathbf{T}_{m}\right]}{L\left(1+e_{m j}\right)^{2}}$

$\mathbf{v}_{m}=\left[\frac{1}{L} \operatorname{tr}\left[\boldsymbol{\Theta}_{m 1} \mathbf{T}_{m}^{2}\right], \ldots, \frac{1}{L} \operatorname{tr}\left[\boldsymbol{\Theta}_{m N} \mathbf{T}_{m}^{2}\right]\right]^{\mathrm{T}}$.

$\mathbf{v}_{m n}=\left[\frac{1}{L} \operatorname{tr}\left[\boldsymbol{\Theta}_{m 1} \mathbf{T}_{m} \boldsymbol{\Theta}_{m n} \mathbf{T}_{m}\right], \ldots, \frac{1}{L} \operatorname{tr}\left[\boldsymbol{\Theta}_{m N} \mathbf{T}_{m} \boldsymbol{\Theta}_{m n} \mathbf{T}_{m}\right]\right]^{\mathrm{T}}$.

Besides, the initial values of $e_{m n} ; \forall n$ to calculate (30) and (31) are set to $e_{m n}^{0}=\frac{1}{\alpha}, n=1, \ldots, N[23]$.

Proof. See Appendix C for derivations.

Remark 7. In order to reduce the amount of overhead exchanged over the fronthaul network, channel estimation is performed locally at each AP and MRT, fpZF and $m R Z F$ precoders are designed based on the local CSI at each AP (17). In this case, pilot signals are not shared over the fronthaul link. Indeed, only channel statistics (large scale parameters which changes slowly [8]) are sent to the CPU for user ordering and power allocation process. Thus, assuming a given system parameters $M, L, N$, and $K$, all three precoders have the same front-hauling overhead. In particular, for each realization of the user locations, the number of $M N K$ statistical parameters need to be exchanged between the $C P U$ and the APs.

\section{Simulation Results}

Herein, we provide simulation results to evaluate the performance of the CF massive MIMO with NOMA or OMA. In NOMA, the same pilot sequence is shared among users within each cluster, but different clusters are assigned mutually orthogonal pilots. In OMA, all the users are assigned with different mutually orthogonal pilots. Therefore, the minimum pilot sequence lengths for NOMA and OMA are $\tau_{\mathrm{NOMA}}=N$ and $\tau_{\mathrm{OMA}}=K N$, respectively. The pre-log factor for NOMA and OMA cases are also defined as $\zeta_{\mathrm{NOMA}}=\left(\tau_{c}-N\right) / \tau_{c}$ and $\zeta_{\mathrm{OMA}}=\left(\tau_{c}-K N\right) / \tau_{c}$, respectively.

In our simulations, the APs are uniformly distributed within an area of size $D \times D \quad \mathrm{~m}^{2}$. Furthermore, users are clustered based on their spatial locations and all the clusters are uniformly distributed at random in the given area (the users in the same cluster are also distributed uniformly at random around the center point of the cluster).

\section{A. Large-Scale Fading Model}

Here, we assume that the large-scale fading coefficient $\beta_{m n k}$ in (1) includes both the path-loss effect and shadowing. Thus, $\beta_{m n k}$ can be wrtten as [8]

$$
\beta_{m n k}=\mathrm{PL}_{m n k}+z_{m n k} \mathrm{~dB},
$$

where $\mathrm{PL}_{m n k}$ is the path loss, $z_{m n k}$ represents the shadow fading generated from a log-normal distribution with standard deviation $\sigma_{\mathrm{sh}}$. A three-slope model is considered for the path loss [42], the path loss exponent equals (i) 3.5 if the distance between the $m$ th AP and the $k$ th user in the $n$th cluster $\left(d_{m n k}\right)$ is greater than $d_{1}$, (ii) equals 2 if $d_{0}<d_{m n k} \leq d_{1}$, and (iii) equals 0 if $d_{m n k} \leq d_{0}$ for some $d_{0}$ and $d_{1}$. When $d_{m n k}>$ $d_{1}$, the Hata-COSTA231 propagation model is employed [8]. Therefore, the path loss can be written as

$$
\mathrm{PL}_{m n k}=\left\{\begin{array}{l}
-\mathcal{L}-35 \log _{10}\left(d_{m n k}\right), \\
-\mathcal{L}-15 \log _{10}\left(d_{1}\right)-20 \log _{10}\left(d_{m n k}\right), \\
d_{0}<d_{m n k} \leq d_{1} \\
-\mathcal{L}-15 \log _{10}\left(d_{1}\right)-20 \log _{10}\left(d_{0}\right), \\
d_{m n k} \leq d_{0}
\end{array}\right.
$$

where

$$
\begin{aligned}
\mathcal{L} & \triangleq 46.3+33.9 \log _{10}(f)-13.82 \log _{10}\left(h_{A P}\right) \\
& -\left(1.1 \log _{10}(f)-0.7\right) h_{u}+\left(1.56 \log _{10}(f)-0.8\right) .
\end{aligned}
$$

In (41), $f$ is the carrier frequency (in $\mathrm{MHz}$ ). Also, $h_{\mathrm{AP}}$ and $h_{u}$ are the AP antenna and user antenna heights (in $\mathrm{m})$, respectively. Note that when $d_{m n k} \leq d_{1}$, there is no shadowing.

\section{B. Parameters and Setup}

The simulation parameters are reported in Table I. The noise variance is given by $\sigma_{w}^{2}=290 \times k_{b} \times$ bandwidth $\times$ noise figure, where $k_{b}$ is the Boltzmann constant. To each cluster, the $m$ th AP allocates power $\frac{p_{t}}{N}$. We further assume that $K=2$; i.e., each cluster has two users, and the total power allocated for the $n$th cluster $p_{n}$ is divided between the two users within a cluster based on a $3: 7$ ratio $(\forall n)$ implying that $\lambda_{n 1}=$ $0.3 \lambda_{n}, \lambda_{n 2}=0.7 \lambda_{n}$. However, these power coefficients are not necessarily optimal and can be further optimized. 
TABLE I

SIMULATION SETTINGS

\begin{tabular}{cccc}
\hline \hline Parameter & Value & Parameter & Value \\
\hline Carrier frequency & $1.9 \mathrm{GHz}$ & $\tau_{c}$ & 56 \\
Bandwidth & $20 \mathrm{MHz}$ & $p_{p}$ & $20 \mathrm{dBm}$ \\
Noise figure & $9 \mathrm{~dB}$ & $p_{t}$ & $23 \mathrm{dBm}$ \\
$D, d_{1}, d_{0}$ & $1000,50,10 \mathrm{~m}$ & $\sigma_{s h}$ & $8 \mathrm{~dB}$ \\
$h_{\mathrm{AP}}, h_{\mathrm{u}}$ & $65,15 \mathrm{~m}$ & $\rho_{n i}$ & 0.1 \\
\hline
\end{tabular}

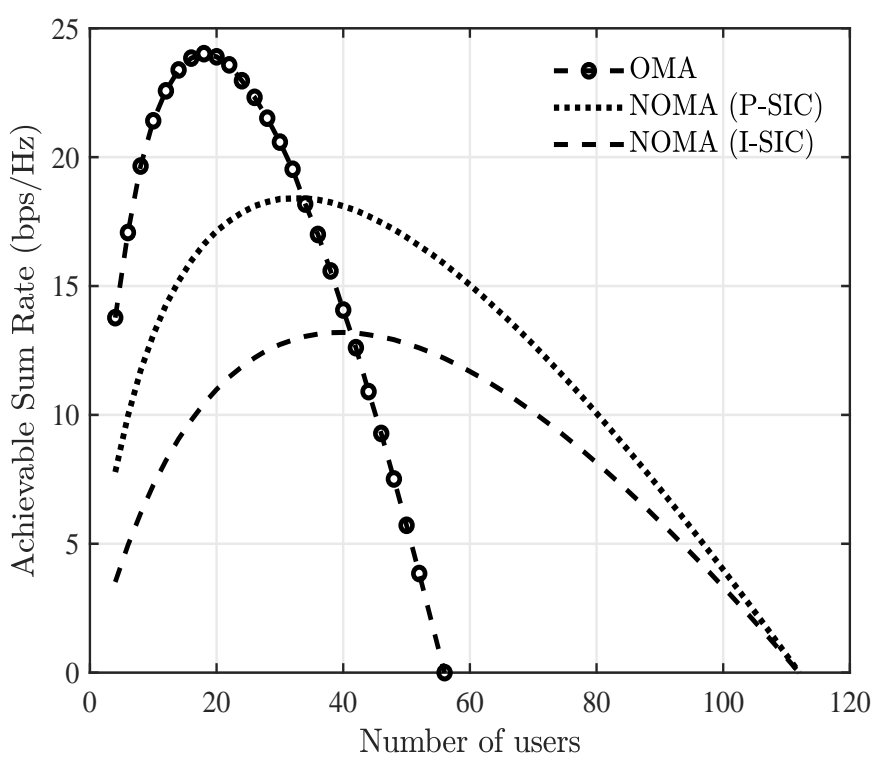

Fig. 2. The achievable sum rate versus the number of users for $25 \mathrm{APs}(\mathrm{M}=$ 25 ) and eight antennas per AP ( $\mathrm{L}=8$ with MRT precoding). P-SIC and I-SIC stand for perfect SIC and imperfect SIC.

\section{Performance Evaluation}

Here, we first compare the performance of the CF massive MIMO-NOMA and OMA systems for MRT, fpZF and mRZF precoding in terms of sum rate given by [43]

$$
\mathcal{R}=\sum_{n=1}^{N} \sum_{k=1}^{K} \mathcal{R}_{n k}
$$

Fig. 2 demonstrates the achievable sum rate (42) of NOMA and OMA systems with MRT precoding as a function of the number of users. We set $\tau_{c}=56$. We observe that the maximum number of users simultaneously served in OMA in the same resource block is $K_{\max }^{\mathrm{OMA}}=56$. In contrast, NOMA doubles this. This is due to the fact that in OMA each user is assigned with an orthogonal pilot while in NOMA same pilot sequence is shared among users within each cluster. For a large number of users, NOMA outperforms OMA; however, with fewer users, NOMA achieves a lower sum rate than OMA due to the effects of intra-cluster pilot contamination and imperfect SIC. We also observe that, SIC imperfection considerably degrades the performance of NOMA; for instance, compared to the perfect SIC, the residual interference caused by imperfect SIC degrades the achievable sum rate by $4.9 \mathrm{bps} / \mathrm{Hz}$ for 40 simultaneously served users.

In Fig. 3, the impact of deploying more antennas at the APs is investigated. As expected, adding more antennas at the APs results in better sum rate, thanks to the array gain.

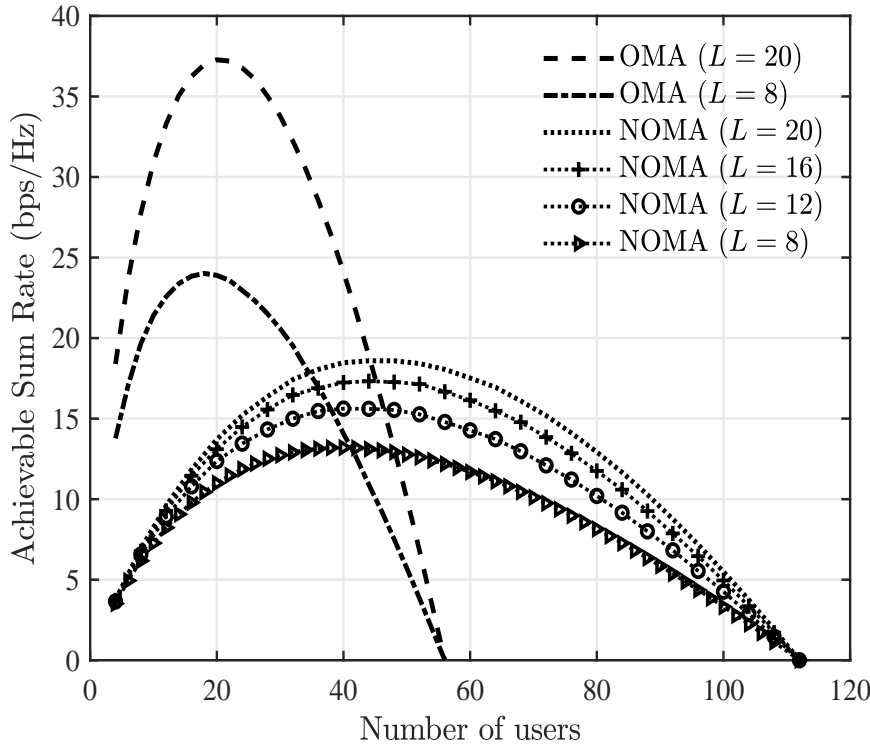

Fig. 3. The achievable sum rate versus the number of users for 25 APs ( $M=25$, MRT, and imperfect SIC).

However, despite OMA, the gain of adding more antennas diminishes for NOMA system since the interference due to the pilot contamination and imperfect SIC proportionally increases as $L$ increases. This observation confirms Remarks 1 and 6

Fig. 4 shows the achievable sum rate of NOMA and OMA systems as a function of the number of users. Here, fpZF precoding and at least $N+1 \leq L$ antennas (24) per AP are considered. We see that the residual interference caused by imperfect SIC significantly degrades the achievable sum rate. More specifically, for 40 users and 60 antennas per AP, the gap between I-SIC and P-SIC curves is about $53 \mathrm{bps} / \mathrm{Hz}$. For imperfect SIC, the gain due to antennas at APs is almost negligible. This is because, for a low number of users (clusters), the SINR converges to (25), where imperfect SIC is the dominant term. Furthermore, as the number of users increases, both the desired power and interference power due to imperfect SIC and pilot contamination increase proportionally to $L-N$.

For the mRZF precoder, the SINR asymptotic $\gamma_{n k}^{\mathrm{mRZF}}$ in 29] must be validated. Thus, Fig. 5 shows the error of the sum rate $\mathcal{R}$ computed based on (29) compared to the ergodic sum rate via simulations of the SINR. Clearly, the SINR asymptotic holds even for small values of $L$ and becomes more accurate as $L$ increases.

Fig. 6 shows the achievable sum rate of NOMA and OMA systems for different number of users with mRZF precoding and finite system dimensions. In this scheme, we simply consider $\alpha=0.8$; however, the optimal value of $\alpha$ that accounts for the CSI imperfection could be further investigated [23], [44]. As expected, with imperfect SIC, the resulting error propagation and intra-cluster pilot contamination limit the system performance. Just as in the case of MRT and fpZF, for a large number of users, NOMA outperforms OMA; however, with a few users, the converse is true.

In Fig. 7 and Fig. 8, we compare the performance of the three precoders for perfect and imperfect SIC, respectively. We observe that, mRZF achieves higher rates than fpZF and MRT, 


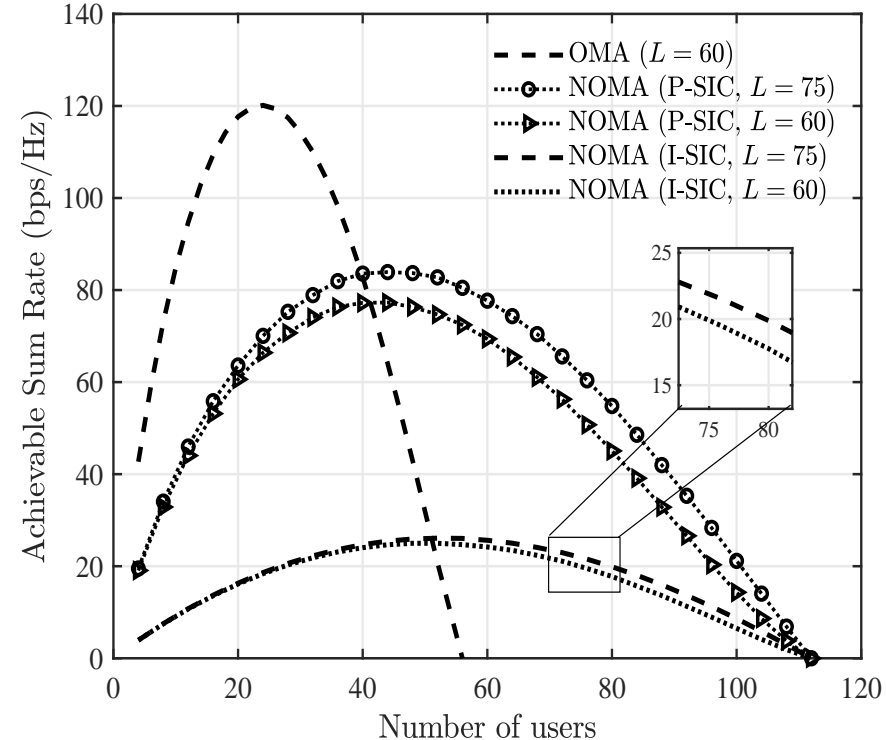

Fig. 4. The achievable sum rate as a function of the number of users for 25 APs (fpZF).

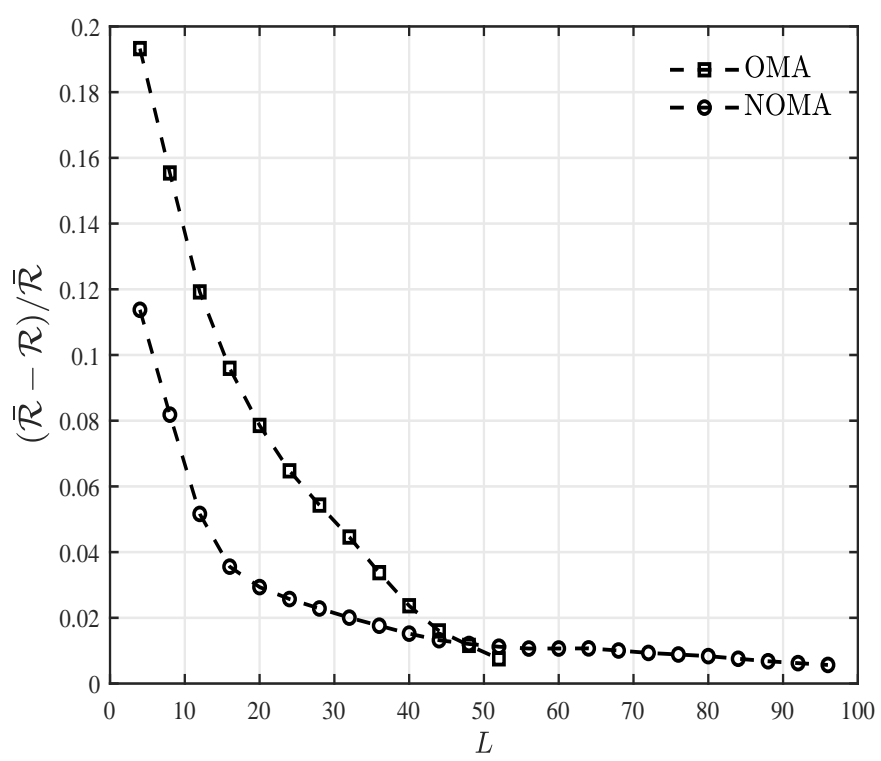

Fig. 5. The mRZF precoder; the relative error of the sum rate $\mathcal{R}$ via (29) compared to the ergodic sum rate; $(\overline{\mathcal{R}}-\mathcal{R}) / \overline{\mathcal{R}}$ versus the number of antennas per AP, $L=K N$, for 25 APs and $\alpha=0.8$.

since it tries to balance the inter-cluster interference mitigation and intra-cluster power enhancement. As well, for perfect SIC, $\mathrm{fpZF}$ and $\mathrm{mRZF}$ outperform MRT as they are able to cancel the inter-cluster interference. Therefore, although fpZF and mRZF have the same front-hauling overhead as MRT, they can achieve higher rates. Furthermore, CF hybrid massive MIMONOMA with either mRZF or fpZF outperforms OMA systems with MRT as twice number of users could be served at the price of a negligible performance loss for a lightly loaded system. We also observe from Fig. 8, with imperfect SIC, MRT and $\mathrm{mRZF}$ perform roughly the same for a large number of users. This is because both fpZF and mRZF sacrifice much of the array gain to suppress the inter-cluster interference;

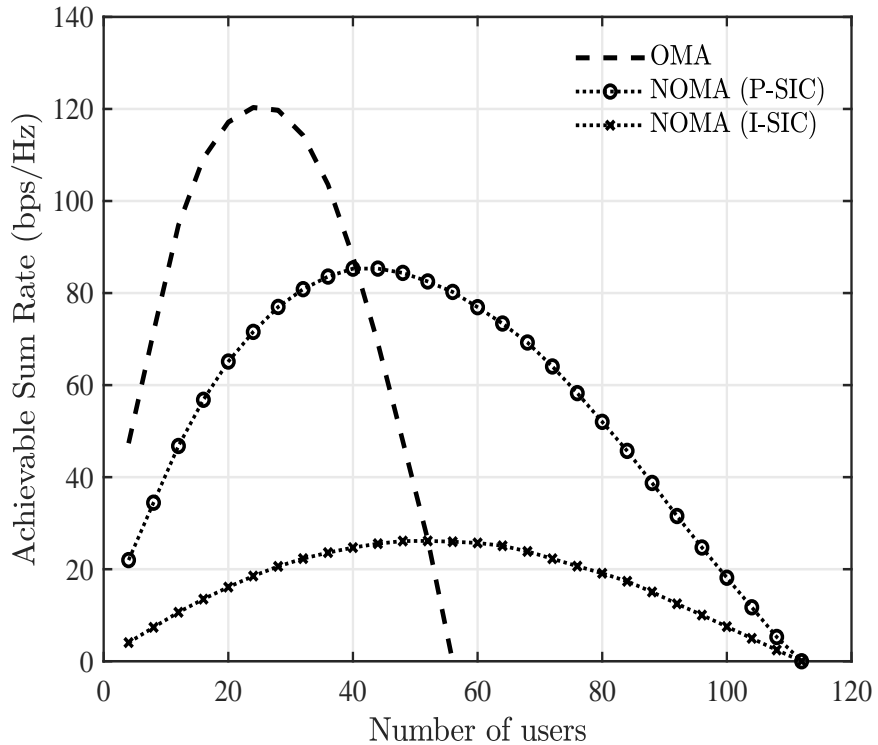

Fig. 6. The achievable sum rate versus the number of users for 25 APs and 60 antennas per AP and mRZF with $\alpha=0.8$.

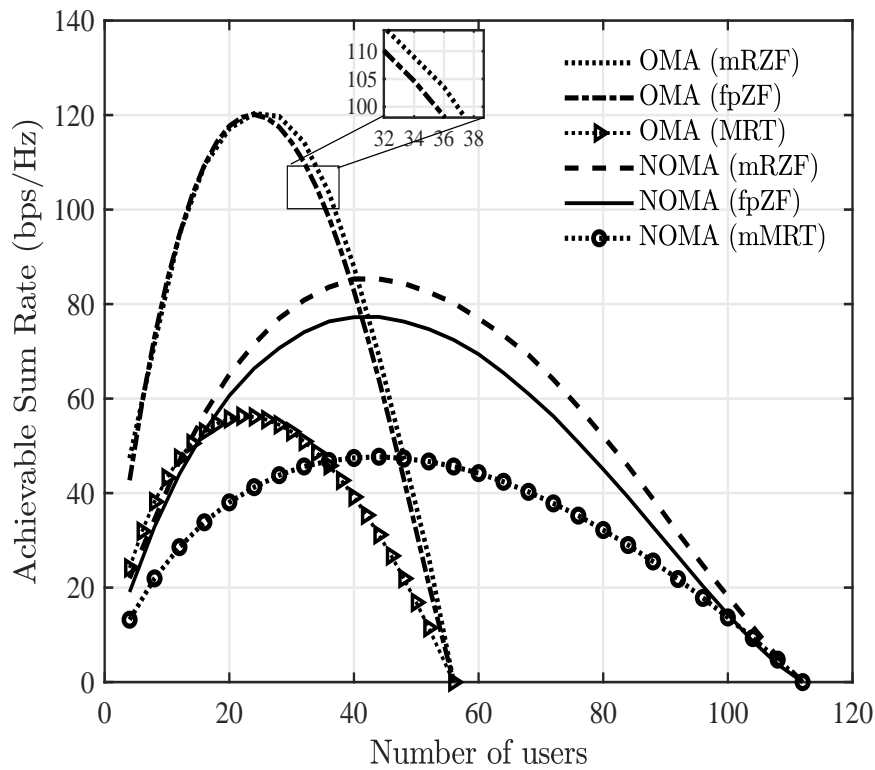

Fig. 7. The achievable sum rate versus the number of users for 25 APs and 60 antennas per AP and perfect SIC.

however mRZF outperforms fpZF since it also considers the desired power. We also note that $\alpha$ may be optimized to enhance the performance of mRZF precoding.

To further investigate these three precoders, we also plot the cumulative distributions of their rates per-cluster in Fig. 9 It shows that the per-cluster rate degrades due to imperfect SIC. In particular, in 90\%-likely performance, all the three precoders behave almost the same and achieve low per-cluster rate of $\sim 1 \mathrm{bps} / \mathrm{Hz}$. In the case of perfect SIC, however, mRZF and $\mathrm{fpZF}$ significantly outperform MRT. Thus, the rate loss due to the imperfect SIC is more considerable for fpZF and mRZF compared to that of MRT. More precisely, the $90 \%$ likely per-cluster rate loss is, respectively, about $1.8 \mathrm{bps} / \mathrm{Hz}$ and $2.2 \mathrm{bps} / \mathrm{Hz}$ for $\mathrm{fpZF}$ and $\mathrm{mRZF}$, which are more than 


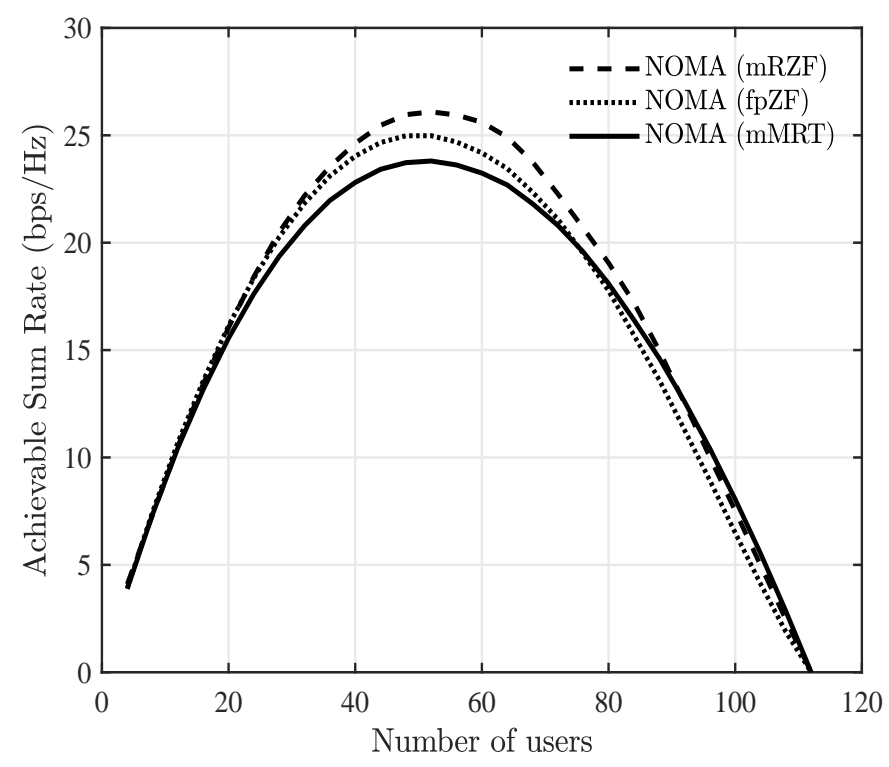

Fig. 8. The achievable sum rate versus the number of users for 25 APs and 60 antennas per AP (Imperfect SIC).

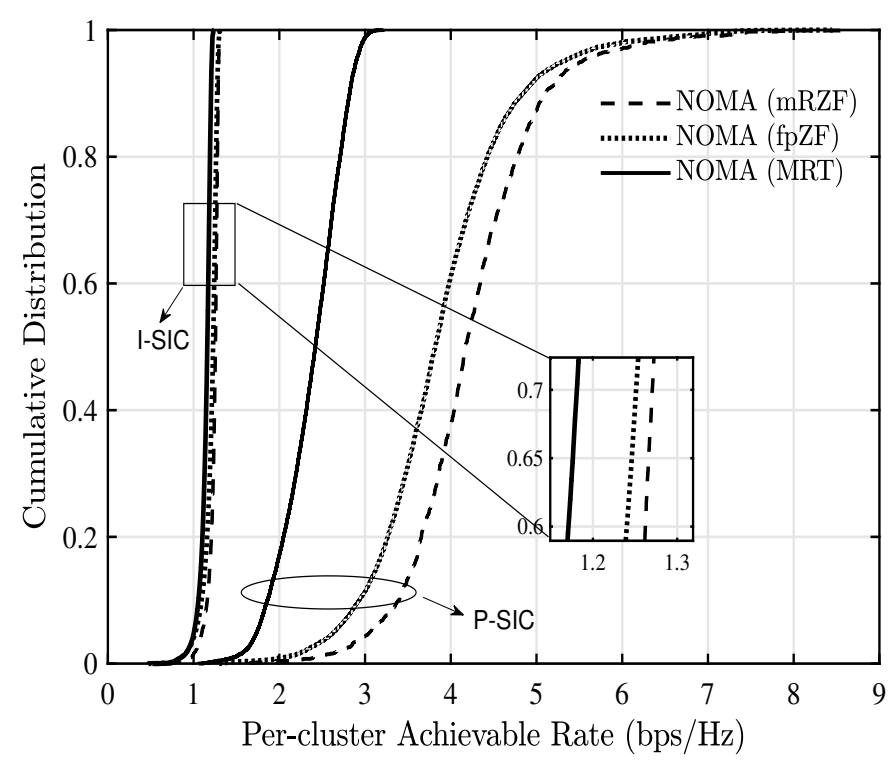

Fig. 9. Cumulative distribution of per-cluster achievable rate for 25 APs and 60 antennas per AP and twenty clusters (NOMA).

twice than that of MRT $(0.8 \mathrm{bps} / \mathrm{Hz})$.

\section{CONCLUSION}

In this paper, we analyzed the achievable rate of three standard precoders for NOMA-aided CF massive MIMO. We thus derived closed-form sum rates for MRT and fpZF by considering joint effects of intra-cluster pilot contamination, inter-cluster interference and imperfect SIC. However, since a closed-form rate analysis is intractable for finite $\mathrm{mRZF}$ precoder, we analyzed the asymptotic regime, where the number of clusters and the number of antennas at each AP grow extremely large while keeping a finite ratio.

We showed that NOMA based CF massive MIMO can support significantly more users compared to the OMA-hybrid at the same time-frequency resources. For a large number of users, the NOMA-hybrid outperforms the OMA-hybrid; however, for a few users, the former achieves lower sum rate than the latter due to the effects of intra-cluster pilot contamination and imperfect SIC. It was further shown that, with perfect SIC, mRZF and fpZF significantly outperforms MRT despite of having the same front-hauling overhead. Finally, CF hybrid massive MIMO-NOMA with either fpZF or mRZF outperforms the OMA systems with MRT.

Future research includes the optimization of $\alpha$ of mRZF precoder, optimal user clustering and power allocations.

\section{APPENDIX A \\ DERIVATION OF $\gamma_{n k}^{\mathrm{MRT}}$ 20,}

By invoking (18) in (19), the MRT precoding vector can be written as

$$
\mathbf{w}_{m n}=\frac{\hat{\mathbf{h}}_{m n k}}{\sqrt{L \theta_{m n k}}} .
$$

We first proceed to compute $\mathbb{E}\left\{\eta_{n k}\right\}$ as follows:

$$
\begin{aligned}
\mathbb{E}\left\{\eta_{n k}\right\} & =\mathbb{E}\left\{\sum_{m=1}^{M} \mathbf{h}_{m n k}^{\mathrm{H}} \mathbf{w}_{m n}\right\} \\
& =\mathbb{E}\left\{\sum_{m=1}^{M}\left(\hat{\mathbf{h}}_{m n k}^{\mathrm{H}}+\boldsymbol{\epsilon}_{m n k}^{\mathrm{H}}\right) \mathbf{w}_{m n}\right\} \\
& =\sum_{m=1}^{M} \sqrt{L \theta_{m n k}} .
\end{aligned}
$$

Since $\mathbf{h}_{m n k}^{\mathrm{H}} \mathbf{w}_{m n}$ are independent for different values of $m$ and the variance of a sum of independent random variables is equal to the sum of the variances, we have

$$
\begin{aligned}
\mathbb{E} & \left\{\left|\left(\eta_{n k}-\mathbb{E}\left\{\eta_{n k}\right\}\right)\right|^{2}\right\} \\
= & \mathbb{E}\left\{\left|\sum_{m=1}^{M} \mathbf{h}_{m n k}^{\mathrm{H}} \mathbf{w}_{m n}-\mathbb{E}\left\{\sum_{m=1}^{M} \mathbf{h}_{m n k}^{\mathrm{H}} \mathbf{w}_{m n}\right\}\right|^{2}\right\} \\
= & \sum_{m=1}^{M} \mathbb{E}\left\{\left|\mathbf{h}_{m n k}^{\mathrm{H}} \mathbf{w}_{m n}\right|^{2}\right\}-\sum_{m=1}^{M}\left|\mathbb{E}\left\{\mathbf{h}_{m n k}^{\mathrm{H}} \mathbf{w}_{m n}\right\}\right|^{2} \\
= & \sum_{m=1}^{M} \mathbb{E}\left\{\left|\left(\hat{\mathbf{h}}_{m n k}^{\mathrm{H}}+\boldsymbol{\epsilon}_{m n k}^{\mathrm{H}}\right) \frac{\hat{\mathbf{h}}_{m n k}}{\sqrt{L \theta_{m n k}}}\right|^{2}\right\}-\sum_{m=1}^{M} L \theta_{m n k} \\
= & \sum_{m=1}^{M} \mathbb{E}\left\{\left|\hat{\mathbf{h}}_{m n k}^{\mathrm{H}} \frac{\hat{\mathbf{h}}_{m n k}}{\sqrt{L \theta_{m n k}}}\right|^{2}\right\}+\sum_{m=1}^{M} \mathbb{E}\left\{\left|\boldsymbol{\epsilon}_{m n k}^{\mathrm{H}} \frac{\hat{\mathbf{h}}_{m n k}}{\sqrt{L \theta_{m n k}}}\right|^{2}\right\} \\
- & \sum_{m=1}^{M} L \theta_{m n k} \\
& \stackrel{(a)}{=}(L+1) \sum_{m=1}^{M} \theta_{m n k}+\sum_{m=1}^{M}\left(\beta_{m n k}-\theta_{m n k}\right)-L \sum_{m=1}^{M} \theta_{m n k} \\
= & \sum_{m=1}^{M} \beta_{m n k},
\end{aligned}
$$

where $(a)$ comes from the fact that, for any vector $\mathbf{z} \sim$ $\mathcal{C N}\left(\mathbf{0}, \theta \mathbf{I}_{L}\right)$, we have [40]

$$
\mathbb{E}\left\{\left|\mathbf{z}^{\mathrm{H}} \mathbf{z}\right|^{2}\right\}=\left(L^{2}+L\right) \theta^{2} .
$$


To find $\mathbb{E}\left\{\left|\eta_{n k}\right|^{2}\right\}$, we use $\operatorname{Var}\{X\}=\mathbb{E}\left\{X^{2}\right\}-(\mathbb{E}\{X\})^{2}$. Thus, we have

$$
\begin{aligned}
\mathbb{E}\left\{\left|\eta_{n k}\right|^{2}\right\} & =\mathbb{E}\left\{\left|\left(\eta_{n k}-\mathbb{E}\left\{\eta_{n k}\right\}\right)\right|^{2}\right\}+\left(\mathbb{E}\left\{\eta_{n k}\right\}\right)^{2} \\
& =\sum_{m=1}^{M} \beta_{m n k}+L\left(\sum_{m=1}^{M} \sqrt{\theta_{m n k}}\right)^{2} .
\end{aligned}
$$

Furthermore,

$$
\begin{aligned}
& \mathbb{E}\left\{\left|\eta_{n^{\prime} k}\right|^{2}\right\}=\mathbb{E}\left\{\left|\sum_{m=1}^{M} \mathbf{h}_{m n k}^{\mathrm{H}} \mathbf{w}_{m n^{\prime}}\right|^{2}\right\} \\
& =\sum_{m=1}^{M} \mathbb{E}\left\{\left|\mathbf{h}_{m n k}^{\mathrm{H}} \mathbf{w}_{m n^{\prime}}\right|^{2}\right\} \\
& +\sum_{m=1}^{M} \sum_{\substack{m^{\prime}=1 \\
m^{\prime} \neq m}}^{M} \mathbb{E}\left\{\left|\mathbf{h}_{m n k}^{\mathrm{H}} \mathbf{w}_{m n^{\prime}}\left(\mathbf{h}_{m^{\prime} n k}^{\mathrm{H}} \mathbf{w}_{m^{\prime} n^{\prime}}\right)\right|\right\} \\
& =\sum_{m=1}^{M} \beta_{m n k},
\end{aligned}
$$

where the second term in the second equality is discarded as $\mathbf{h}_{m n k}^{\mathrm{H}} \mathbf{w}_{m n^{\prime}}(\forall m \in 1, \ldots, M)$ are independent of each other.

Moreover, since $\mathbb{E}\left\{s_{n i}^{*} \hat{s}_{n i}\right\}=\mathbb{E}\left\{s_{n i} \hat{s}_{n i}^{*}\right\}=\rho_{n i}$, we have

$$
\begin{aligned}
\mathbb{E}\left\{\left|\eta_{n k} s_{n i}-\mathbb{E}\left\{\eta_{n k}\right\} \hat{s}_{n i}\right|^{2}\right\} & =\mathbb{E}\left\{\left|\eta_{n k}\right|^{2}\right\} \\
& +\left(1-2 \rho_{n i}\right) \mathbb{E}^{2}\left\{\eta_{n k}\right\}
\end{aligned}
$$

Finally, by substituting the above formulations into (15), $\gamma_{n k}^{\mathrm{MRT}}$ can be obtained as 20.

\section{APPENDIX B}

DERIVATION OF $\gamma_{n k}^{\mathrm{ZF}} 24$

For fpZF precoding, we first compute $\mathbb{E}\left\{\eta_{n k}\right\}$ as follows:

$$
\begin{aligned}
\mathbb{E}\left\{\eta_{n k}\right\} & =\mathbb{E}\left\{\sum_{m=1}^{M} \mathbf{h}_{m n k}^{\mathrm{H}} \mathbf{w}_{m n}\right\} \\
& =\mathbb{E}\left\{\sum_{m=1}^{M}\left(\hat{\mathbf{h}}_{m n k}^{\mathrm{H}}+\boldsymbol{\epsilon}_{m n k}^{\mathrm{H}}\right) \mathbf{w}_{m n}\right\} \\
& =\sum_{m=1}^{M} \sqrt{(L-N) \theta_{m n k}} .
\end{aligned}
$$

In the denominator, we have

$$
\mathbb{E}\left\{\left|\left(\eta_{n k}-\mathbb{E}\left\{\eta_{n k}\right\}\right)\right|^{2}\right\}=\mathbb{E}\left\{\left|\eta_{n k}\right|^{2}\right\}-\mathbb{E}^{2}\left\{\eta_{n k}\right\}
$$

Referring to (49) and (51), we just need to calculate $\mathbb{E}\left\{\left|\eta_{n^{\prime} k}\right|^{2}\right\}, \forall n^{\prime}$. Employing the result in 23, we have

$\mathbb{E}\left\{\left|\eta_{n^{\prime} k}\right|^{2}\right\}=\mathbb{E}\left\{\left|\sum_{m=1}^{M} \mathbf{h}_{m n k}^{\mathrm{H}} \mathbf{w}_{m n^{\prime}}\right|^{2}\right\}$
$=\mathbb{E}\left\{\left|\sum_{m=1}^{M}\left(\hat{\mathbf{h}}_{m n k}^{\mathrm{H}}+\boldsymbol{\epsilon}_{m n k}^{\mathrm{H}}\right) \mathbf{w}_{m n^{\prime}}\right|^{2}\right\}$

$=\left\{\begin{array}{lr}(L-N)\left(\sum_{m=1}^{M} \sqrt{\theta_{m n k}}\right)^{2}+\sum_{m=1}^{M}\left(\beta_{m n k}-\theta_{m n k}\right), & n=n^{\prime} \\ \sum_{m=1}^{M}\left(\beta_{m n k}-\theta_{m n k}\right), & n \neq n^{\prime}\end{array}\right.$

By substituting (50) and (52) into (15), $\gamma_{n k}^{\mathrm{fpZF}}$ is in (24).

\section{APPENDIX C \\ DERIVATION OF $\gamma_{n k}^{\mathrm{mRZF}}$ 29)}

To derive SINR $\gamma_{n k}^{\mathrm{mRZF}}$, we need the following terms:

- Desired signal power

$$
p_{d}=p_{n k}\left(\sum_{m=1}^{M} \mathbb{E}\left\{\mathbf{h}_{m n k}^{\mathrm{H}} \mathbf{w}_{m n}\right\}\right)^{2}
$$

- Variance of the beamforming gain uncertainty

$$
p_{I 1}=p_{n k}\left(\sum_{m=1}^{M} \mathbb{E}\left\{\left|\mathbf{h}_{m n k}^{\mathrm{H}} \mathbf{w}_{m n}\right|^{2}\right\}-\sum_{m=1}^{M}\left|\mathbb{E}\left\{\mathbf{h}_{m n k}^{\mathrm{H}} \mathbf{w}_{m n}\right\}\right|^{2}\right) \text {. }
$$

- Inter-cluster interference

$$
p_{I 2}=\sum_{\substack{n^{\prime}=1 \\ n^{\prime} \neq n}}^{N} p_{n^{\prime}} \mathbb{E}\left\{\left|\sum_{m=1}^{M} \mathbf{h}_{m n k}^{\mathrm{H}} \mathbf{w}_{m n^{\prime}}\right|^{2}\right\}
$$

In order to calculate the intra-cluster interference due to the pilot contamination and imperfect SIC, we need $\mathbb{E}\left\{\left|\sum_{m=1}^{M} \mathbf{h}_{m n k}^{\mathrm{H}} \mathbf{w}_{m n}\right|^{2}\right\}$, which can be derived by exploiting (54) and the definition of the variance.

We now proceed to calculate (53), 54 and (55) when $L, N \rightarrow \infty$ while keeping a finite ratio.

Let

$$
\overline{\mathbf{h}}_{m n}=\sqrt{L} \Theta_{m n}^{1 / 2} \overline{\mathbf{g}}_{m n},
$$

where $\overline{\mathbf{g}}_{m n}$ has i.i.d complex entries with zero mean and variance of $\frac{1}{L} \quad\left(\overline{\mathbf{g}}_{m n} \sim \mathcal{C N}\left(\mathbf{0}, \frac{1}{L} \mathbf{I}_{L}\right)\right)$ and $\boldsymbol{\Theta}_{m n}=$ $\left(1+\tau p_{p} \sum_{k=1}^{k} \beta_{m n k}\right) \mathbf{I}_{L}$. Also, assume that $\boldsymbol{\Theta}_{m n} ; \forall m, n$ and $\frac{1}{L} \overline{\mathbf{H}}_{m} \overline{\mathbf{H}}_{m}^{\mathrm{H}}=\sum_{n=1}^{N} \boldsymbol{\Theta}_{m n}^{1 / 2} \overline{\mathbf{g}}_{m n} \overline{\mathbf{g}}_{m n}^{\mathrm{H}} \boldsymbol{\Theta}_{m n}^{1 / 2} ; \forall m$ have uniformly bounded spectral norms [23]. Moreover, we define

$$
\begin{gathered}
\boldsymbol{\Sigma}_{m} \triangleq\left(\overline{\mathbf{H}}_{m} \overline{\mathbf{H}}_{m}^{\mathrm{H}}+L \alpha \mathbf{I}_{L}\right), \\
\boldsymbol{\Sigma}_{m n} \triangleq\left(\overline{\mathbf{H}}_{m n} \overline{\mathbf{H}}_{m n}^{\mathrm{H}}+L \alpha \mathbf{I}_{L}\right),
\end{gathered}
$$




$$
\begin{aligned}
\psi_{m n} & \triangleq \mathbb{E}\left\{\left\|\left(\overline{\mathbf{H}}_{m} \overline{\mathbf{H}}_{m}^{\mathrm{H}}+L \alpha \mathbf{I}_{L}\right)^{-1} \overline{\mathbf{h}}_{m n}\right\|^{2}\right\} \\
& =\mathbb{E}\left\{\overline{\mathbf{h}}_{m n}^{\mathrm{H}} \boldsymbol{\Sigma}_{m}^{-2} \overline{\mathbf{h}}_{m n}\right\} .
\end{aligned}
$$

where $\overline{\mathbf{H}}_{m n}$ is equal to $\overline{\mathbf{H}}_{m}$ with the $n$th column removed.

- Desired signal power

Regarding (53), we derive $\mathbb{E}\left\{\mathbf{h}_{m n k} \mathbf{w}_{m n}\right\}$ as follows:

$$
\begin{aligned}
& \mathbb{E}\left\{\mathbf{h}_{m n k} \mathbf{w}_{m n}\right\} \\
& =\frac{1}{\sqrt{\psi_{m n}}} \mathbb{E}\left\{\mathbf{h}_{m n k}^{\mathrm{H}} \boldsymbol{\Sigma}_{m}^{-1} \overline{\mathbf{h}}_{m n}\right\} \\
& \stackrel{(a)}{=} \frac{1}{\sqrt{\psi_{m n}}} \mathbb{E}\left\{\frac{\mathbf{h}_{m n k}^{\mathrm{H}} \boldsymbol{\Sigma}_{m n}^{-1} \overline{\mathbf{h}}_{m n}}{1+\overline{\mathbf{h}}_{m n}^{\mathrm{H}} \boldsymbol{\Sigma}_{m n}^{-1} \overline{\mathbf{h}}_{m n}}\right\} \\
& =\frac{1}{\sqrt{\psi_{m n}}} \mathbb{E}\left\{\frac{\left(c_{m n k} \overline{\mathbf{h}}_{m n}+\boldsymbol{\epsilon}_{m n k}\right)^{\mathrm{H}} \boldsymbol{\Sigma}_{m n}^{-1} \overline{\mathbf{h}}_{m n}}{1+\overline{\mathbf{h}}_{m n}^{\mathrm{H}} \boldsymbol{\Sigma}_{m n}^{-1} \overline{\mathbf{h}}_{m n}}\right\} \\
& =\frac{1}{\sqrt{\psi_{m n}}} \mathbb{E}\left\{\frac{c_{m n k} \overline{\mathbf{h}}_{m n}^{\mathrm{H}} \boldsymbol{\Sigma}_{m n}^{-1} \overline{\mathbf{h}}_{m n}}{1+\overline{\mathbf{h}}_{m n}^{\mathrm{H}} \boldsymbol{\Sigma}_{m n}^{-1} \overline{\mathbf{h}}_{m n}}+\frac{\boldsymbol{\epsilon}_{m n k}^{\mathrm{H}} \boldsymbol{\Sigma}_{m n}^{-1} \overline{\mathbf{h}}_{m n}}{1+\overline{\mathbf{h}}_{m n}^{\mathrm{H}} \boldsymbol{\Sigma}_{m n}^{-1} \overline{\mathbf{h}}_{m n}}\right\},
\end{aligned}
$$

where $(a)$ comes from the matrix inversion Lemma [23], [45]. In order to solve (60), we use the following Lemma.

Lemma 1. Let $\mathbf{A} \in \mathcal{C}^{L \times L}$ and $\mathbf{x}, \mathbf{y} \sim \mathcal{C N}\left(\mathbf{0}, \frac{1}{L} \mathbf{I}_{L}\right)$. Assume that $\mathbf{A}$ has uniformly bounded spectral norm (with respect to $L$ ) and that $\mathbf{x}$ and $\mathbf{y}$ are mutually independent and independent of $\mathbf{A}$. Now, as $L \longrightarrow \infty$,

$$
\text { a. } \mathbf{x}^{\mathrm{H}} \mathbf{A x} \stackrel{\text { a.s. }}{\rightarrow} \frac{1}{L} \operatorname{tr}[\mathbf{A}], \text { b. } \mathbf{x}^{\mathrm{H}} \mathbf{A y} \stackrel{\text { a.s. }}{\rightarrow} 0,
$$

where $\stackrel{a . s .}{\rightarrow}$ denotes almost sure convergence as $L \longrightarrow \infty$. By substituting $\overline{\mathbf{h}}_{m n}$ with 56 and applying Lemma 1 a and Theorem 2 of [23] to the first term of [60), we have

$$
\begin{aligned}
\frac{c_{m n k} \overline{\mathbf{h}}_{m n}^{\mathrm{H}} \boldsymbol{\Sigma}_{m n}^{-1} \overline{\mathbf{h}}_{m n}}{1+\overline{\mathbf{h}}_{m n}^{\mathrm{H}} \boldsymbol{\Sigma}_{m n}^{-1} \overline{\mathbf{h}}_{m n}} & =\frac{c_{m n k} L \overline{\mathbf{g}}_{m n}^{\mathrm{H}} \boldsymbol{\Theta}_{m n}^{1 / 2} \boldsymbol{\Sigma}_{m n}^{-1} \boldsymbol{\Theta}_{m n}^{1 / 2} \overline{\mathbf{g}}_{m n}}{1+L \overline{\mathbf{g}}_{m n}^{\mathrm{H}} \boldsymbol{\Theta}_{m n}^{1 / 2} \boldsymbol{\Sigma}_{m n}^{-1} \boldsymbol{\Theta}_{m n}^{1 / 2} \overline{\mathbf{g}}_{m n}} \\
& =\frac{c_{m n k} \overline{\mathbf{g}}_{m n}^{\mathrm{H}} \boldsymbol{\Theta}_{m n}^{1 / 2} \mathbf{C}_{m n}^{-1} \boldsymbol{\Theta}_{m n}^{1 / 2} \overline{\mathbf{g}}_{m n}}{1+\overline{\mathbf{g}}_{m n}^{\mathrm{H}} \boldsymbol{\Theta}_{m n}^{1 / 2} \mathbf{C}_{m n}^{-1} \boldsymbol{\Theta}_{m n}^{1 / 2} \overline{\mathbf{g}}_{m n}} \\
& \stackrel{a . s .}{\rightarrow} \frac{c_{m n k} e_{m n}^{o}}{1+e_{m n}^{o}},
\end{aligned}
$$

where $\mathbf{C}_{m n}=\boldsymbol{\Gamma}_{m n}+\alpha \mathbf{I}_{L}$ with $\boldsymbol{\Gamma}_{m n}=\frac{1}{L} \overline{\mathbf{H}}_{m n} \overline{\mathbf{H}}_{m n}^{\mathrm{H}}$. Besides, $e_{m n}^{o}$ is given in (30). Similarly, Since, $\hat{\mathbf{h}}_{m n k}=$ $c_{m n k} \overline{\mathbf{h}}_{m n}$ is independent of $\boldsymbol{\epsilon}_{m n k}$, by applying Lemma [1. $b$ to the second term of 60, we have

$$
\boldsymbol{\epsilon}_{m n k}^{\mathrm{H}} \boldsymbol{\Sigma}_{m n}^{-1} \overline{\mathbf{h}}_{m n} \stackrel{\text { a.s. }}{\rightarrow} 0 .
$$

Regarding (59), to find the value of $\psi_{m n}$, we need to calculate $\mathbb{E}\left\{\overline{\mathbf{h}}_{m n}^{\mathrm{H}} \boldsymbol{\Sigma}_{m}^{-2} \overline{\mathbf{h}}_{m n}\right\}$.

By employing matrix inversion Lemma, Theorems 1 and 2 of [23], we obtain

$$
\begin{aligned}
& \overline{\mathbf{h}}_{m n}^{\mathrm{H}} \boldsymbol{\Sigma}_{m}^{-2} \overline{\mathbf{h}}_{m n} \stackrel{(a)}{=} \frac{1}{L} \frac{\overline{\mathbf{g}}_{m n}^{\mathrm{H}} \boldsymbol{\Theta}_{m n}^{1 / 2} \mathbf{C}_{m n}^{-2} \boldsymbol{\Theta}_{m n}^{1 / 2} \overline{\mathbf{g}}_{m n}}{\left(1+\overline{\mathbf{g}}_{m n}^{\mathrm{H}} \boldsymbol{\Theta}_{m n}^{1 / 2} \mathbf{C}_{m n}^{-1} \boldsymbol{\Theta}_{m n}^{1 / 2} \overline{\mathbf{g}}_{m n}\right)^{2}} \\
& \stackrel{\text { a.s. }}{\rightarrow} \frac{1}{L} \frac{\frac{1}{L} \operatorname{tr}\left[\boldsymbol{\Theta}_{m n} \mathbf{C}_{m n}^{-2}\right]}{\left(1+\frac{1}{L} \operatorname{tr}\left[\mathbf{\Theta}_{m n} \mathbf{C}_{m n}^{-1}\right]\right)^{2}} \\
& \stackrel{\text { a.s. }}{\rightarrow} \frac{1}{L} \frac{\frac{1}{L} \operatorname{tr}\left[\boldsymbol{\Theta}_{m n} \mathbf{T}_{m}^{\prime}\right]}{\left(1+\frac{1}{L} \operatorname{tr}\left[\boldsymbol{\Theta}_{m n} \mathbf{T}_{m}\right]\right)^{2}},
\end{aligned}
$$

where $(a)$ comes from the matrix inversion Lemma which is applied twice, $\mathbf{T}_{m n}$ is defined in (31) and

$$
\mathbf{T}_{m}^{\prime}=\mathbf{T}_{m}\left[\frac{1}{L} \sum_{j=1}^{N} \frac{\boldsymbol{\Theta}_{m j} e_{m j}^{\prime}}{\left(1+e_{m j}\right)^{2}}+\mathbf{I}_{L}\right] \mathbf{T}_{m}
$$

where

$$
e_{m j}^{\prime}=\frac{1}{L} \operatorname{tr}\left[\boldsymbol{\Theta}_{m j} \mathbf{T}_{m}^{\prime}\right] .
$$

By employing Theorem 2 of [23], we finally have

$$
\overline{\mathbf{h}}_{m n}^{\mathrm{H}} \boldsymbol{\Sigma}_{m}^{-2} \overline{\mathbf{h}}_{m n} \stackrel{a . s .}{\rightarrow} \psi_{m n}^{o},
$$

where $\psi_{m}^{o}$ is given in 32). Therefore, by invoking 62 and (67) in (53), desired signal power can be written as

$$
p_{d} \stackrel{a . s .}{\rightarrow} p_{n, k}\left(\sum_{m=1}^{M} \frac{1}{\sqrt{\psi_{m n}^{o}}} \frac{c_{m n k} e_{m n}^{o}}{1+e_{m n}^{o}}\right)^{2} .
$$

- Variance of the beamforming gain uncertainty Regarding (54), We need to calculate the term $\left(\mathbb{E}\left\{\left|\mathbf{h}_{m n k}^{\mathrm{H}} \mathbf{w}_{m n}\right|^{2}\right\}\right)$. By employing (60) and 62), we have

$$
\left|\mathbf{h}_{m n k}^{\mathrm{H}} \mathbf{w}_{m n}\right|^{2} \stackrel{\text { a.s. }}{\rightarrow} \frac{1}{\psi_{m n}^{o}} \frac{c_{m n k}^{2}\left(e_{m n}^{o}\right)^{2}}{\left(1+e_{m n}^{o}\right)^{2}} .
$$

Substituting 62, 67) and 69 in 54, we obtain

$$
\begin{aligned}
& p_{I 1} \stackrel{a . s .}{\rightarrow} p_{n k}\left(\sum_{m=1}^{M} \frac{1}{\psi_{m n}^{o}} \frac{c_{m n k}^{2}\left(e_{m n}^{o}\right)^{2}}{\left(1+e_{m n}^{o}\right)^{2}}-\sum_{m=1}^{M} \frac{1}{\psi_{m n}^{o}} \frac{c_{m n k}^{2}\left(e_{m n}^{o}\right)^{2}}{\left(1+e_{m n}^{o}\right)^{2}}\right) \\
& \stackrel{\text { a.s. }}{\rightarrow} 0 \text {. }
\end{aligned}
$$

We also find

$$
\mathbb{E}\left\{\left|\sum_{m=1}^{M} \mathbf{h}_{m n k}^{\mathrm{H}} \mathbf{w}_{m n}\right|^{2}\right\} \stackrel{\text { a.s. }}{\rightarrow}\left(\sum_{m=1}^{M} \frac{1}{\sqrt{\psi_{m n}^{o}}} \frac{c_{m n k} e_{m n}^{o}}{1+e_{m n}^{o}}\right)^{2} .
$$

- Inter-cluster interference

In order to derive [55], we define

$\mathbf{P}_{m n} \triangleq \operatorname{Diag}\left(\frac{p_{1}}{\psi_{m 1}^{o}}, \ldots, \frac{p_{n-1}}{\psi_{m(n-1)}^{o}}, \frac{p_{n+1}}{\psi_{m(n+1)}^{o}}, \ldots, \frac{p_{N}}{\psi_{m N}^{o}}\right)$.

By applying the matrix inversion Lemma, Lemma 1 and (72), (55) can be written as

$$
\begin{aligned}
p_{I 2} & =\sum_{\substack{n^{\prime}=1 \\
n^{\prime} \neq n}}^{N} p_{n^{\prime}} \sum_{m=1}^{M} \mathbb{E}\left\{\left|\mathbf{h}_{m n k}^{\mathrm{H}} \mathbf{w}_{m n^{\prime}}\right|^{2}\right\} \\
& =\sum_{m=1}^{M} \mathbb{E}\left\{\mathbf{h}_{m n k}^{\mathrm{H}} \boldsymbol{\Sigma}_{m}^{-1} \overline{\mathbf{H}}_{m n} \mathbf{P}_{m n} \overline{\mathbf{H}}_{m n}^{\mathrm{H}} \boldsymbol{\Sigma}_{m}^{-1} \mathbf{h}_{m n k}\right\} .
\end{aligned}
$$

Since $\mathbf{h}_{m n k}=\hat{\mathbf{h}}_{m n k}+\boldsymbol{\epsilon}_{m n k}$ and $\hat{\mathbf{h}}_{m n k}=c_{m n k} \overline{\mathbf{h}}_{m n}$, we find that the estimated channels of the users in the same cluster are parallel. Accordingly, $\mathbf{h}_{m n k}$ can be written as

$$
\mathbf{h}_{m n k}=\sqrt{L} \boldsymbol{\Theta}_{m n}^{1 / 2}\left[c_{m n k} \overline{\mathbf{g}}_{m n}+a_{m n k} \hat{\mathbf{g}}_{m n k}\right],
$$


where $a_{m n k}=\sqrt{\frac{\beta_{m n k}-\theta_{m n k}}{1+\tau p_{p} \sum_{k=1}^{K} \beta_{m n k}}}$ and $\hat{\mathbf{g}}_{m n k} \sim$ $\mathcal{C N}\left(\mathbf{0}, \frac{1}{L} \mathbf{I}_{L}\right)$ is independent of $\overline{\mathbf{g}}_{m n}$. By substituting (74) in $(73)$, we find

$$
\begin{aligned}
& \mathbf{h}_{m n k}^{\mathrm{H}} \boldsymbol{\Sigma}_{m}^{-1} \overline{\mathbf{H}}_{m n} \mathbf{P}_{m n} \overline{\mathbf{H}}_{m n}^{\mathrm{H}} \boldsymbol{\Sigma}_{m}^{-1} \mathbf{h}_{m n k} \\
& =\frac{1}{L} c_{m n k}^{2} \overline{\mathbf{g}}_{m n}^{\mathrm{H}} \boldsymbol{\Theta}_{m n}^{1 / 2} \mathbf{C}_{m}^{-1} \overline{\mathbf{H}}_{m n} \mathbf{P}_{m n} \overline{\mathbf{H}}_{m n}^{\mathrm{H}} \mathbf{C}_{m}^{-1} \mathbf{\Theta}_{m n}^{1 / 2} \overline{\mathbf{g}}_{m n} \\
& +\frac{1}{L} a_{m n k}^{2} \hat{\mathbf{g}}_{m n k}^{\mathrm{H}} \boldsymbol{\Theta}_{m n}^{1 / 2} \mathbf{C}_{m}^{-1} \overline{\mathbf{H}}_{m n} \mathbf{P}_{m n} \overline{\mathbf{H}}_{m n}^{\mathrm{H}} \mathbf{C}_{m}^{-1} \mathbf{\Theta}_{m n}^{1 / 2} \hat{\mathbf{g}}_{m n k} \\
& +\frac{1}{L} c_{m n k} a_{m n k} \overline{\mathbf{g}}_{m n}^{\mathrm{H}} \boldsymbol{\Theta}_{m n}^{1 / 2} \mathbf{C}_{m}^{-1} \overline{\mathbf{H}}_{m n} \mathbf{P}_{m n} \overline{\mathbf{H}}_{m n}^{\mathrm{H}} \mathbf{C}_{m}^{-1} \boldsymbol{\Theta}_{m n}^{1 / 2} \hat{\mathbf{g}}_{m n k} \\
& +\frac{1}{L} c_{m n k} a_{m n k} \hat{\mathbf{g}}_{m n k}^{\mathrm{H}} \boldsymbol{\Theta}_{m n}^{1 / 2} \mathbf{C}_{m}^{-1} \overline{\mathbf{H}}_{m n} \mathbf{P}_{m n} \overline{\mathbf{H}}_{m n}^{\mathrm{H}} \mathbf{C}_{m}^{-1} \mathbf{\Theta}_{m n}^{1 / 2} \overline{\mathbf{g}}_{m n}
\end{aligned}
$$

where $\mathbf{C}_{m}=\boldsymbol{\Gamma}_{m}+\alpha \mathbf{I}_{L}$ with $\boldsymbol{\Gamma}_{m}=\frac{1}{L} \overline{\mathbf{H}}_{m} \overline{\mathbf{H}}_{m}^{\mathrm{H}}$. The equation in (75) can be further written as (76). Employing Lemma 2 of $[\overline{23}], \mathbf{C}_{m}^{-1}-\mathbf{C}_{m n}^{-1}=-\mathbf{C}_{m}^{-1}\left(\mathbf{C}_{m}-\mathbf{C}_{m n}\right) \mathbf{C}_{m n}^{-1}$ with $\mathbf{C}_{m}-\mathbf{C}_{m n}=\boldsymbol{\Theta}_{m n}^{1 / 2} \overline{\mathbf{g}}_{m n} \overline{\mathbf{g}}_{m n}^{\mathrm{H}} \boldsymbol{\Theta}_{m n}^{1 / 2}$. Then, 76 can be written as 77) shown at the top of the next page, where $\mathbf{B}_{m n}=\boldsymbol{\Theta}_{m n}^{1 / 2} \mathbf{C}_{m n}^{-1} \overline{\mathbf{H}}_{m n} \mathbf{P}_{m n} \overline{\mathbf{H}}_{m n}^{\mathrm{H}} \mathbf{C}_{m}^{-1} \mathbf{\Theta}_{m n}^{1 / 2}$ and $\mathbf{A}_{m n}=$ $\mathbf{\Theta}_{m n}^{1 / 2} \mathbf{C}_{m}^{-1} \boldsymbol{\Theta}_{m n}^{1 / 2}$. Then, by applying Lemma 7 of [23] to each quadratic form in (77), we obtain

$$
\begin{aligned}
& \text { a. } \overline{\mathbf{g}}_{m n}^{\mathrm{H}} \mathbf{A}_{m n} \overline{\mathbf{g}}_{m n} \stackrel{\text { a.s. }}{\rightarrow} \frac{u_{m n}}{1+u_{m n}}, \\
& \text { b. } \hat{\mathbf{g}}_{m n k}^{\mathrm{H}} \mathbf{A}_{m n} \overline{\mathbf{g}}_{m n} \stackrel{\text { a.s. }}{\rightarrow} 0, \\
& \text { c. } \overline{\mathbf{g}}_{m n}^{\mathrm{H}} \mathbf{B}_{m n} \overline{\mathbf{g}}_{m n} \stackrel{\text { a.s. }}{\rightarrow} \frac{u_{m n}^{\prime}}{1+u_{m n}}, \\
& \text { d. } \hat{\mathbf{g}}_{m n k}^{\mathrm{H}} \mathbf{B}_{m n} \hat{\mathbf{g}}_{m n k} \stackrel{\text { a.s. }}{\rightarrow} u_{m n}^{\prime}, \\
& \text { e. } \hat{\mathbf{g}}_{m n k}^{\mathrm{H}} \mathbf{B}_{m n} \overline{\mathbf{g}}_{m n} \stackrel{\text { a.s. }}{\rightarrow} 0,
\end{aligned}
$$

where $u_{m n}=\frac{1}{L} \operatorname{tr}\left[\boldsymbol{\Theta}_{m n} \mathbf{C}_{m n}^{-1}\right]$ and $u_{m n}^{\prime}=$ $\frac{1}{L} \operatorname{tr}\left[\mathbf{P}_{m n} \overline{\mathbf{H}}_{m n}^{\mathrm{H}} \mathbf{C}_{m n}^{-1} \boldsymbol{\Theta}_{m n} \mathbf{C}_{m n}^{-1} \overline{\mathbf{H}}_{m n}\right]$. By substituting (78) in (77), we obtain

$$
\begin{aligned}
& \mathbf{h}_{m n k}^{\mathrm{H}} \boldsymbol{\Sigma}_{m}^{-1} \overline{\mathbf{H}}_{m n} \mathbf{P}_{m n} \overline{\mathbf{H}}_{m n}^{\mathrm{H}} \boldsymbol{\Sigma}_{m}^{-1} \mathbf{h}_{m n k} \\
& \stackrel{\text { a.s. }}{\rightarrow} c_{m n k}^{2}\left(\frac{1}{L} \frac{u_{m n}^{\prime}}{1+u_{m n}}-\frac{1}{L} \frac{u_{m n} u_{m n}^{\prime}}{\left(1+u_{m n}\right)^{2}}\right) \\
& +a_{m n k}^{2}\left(\frac{1}{L} u_{m n}^{\prime}\right) \\
& \stackrel{\text { a.s. }}{\rightarrow} c_{m n k}^{2} \frac{1}{L} \frac{u_{m n}^{\prime}}{\left(1+u_{m n}\right)^{2}}+a_{m n k}^{2} \frac{1}{L} u_{m n}^{\prime},
\end{aligned}
$$

By employing Lemma 6 of [23], we have

$$
\begin{aligned}
& u_{m n} \stackrel{\text { a.s. }}{\rightarrow} \frac{1}{L} \operatorname{tr}\left[\boldsymbol{\Theta}_{m n} \mathbf{C}_{m}^{-1}\right] \\
& \stackrel{\text { a.s. }}{\rightarrow} e_{m n}^{o} \\
& \frac{1}{L} u_{m n}^{\prime} \stackrel{\text { a.s. }}{\rightarrow} \bar{\Upsilon}_{m n},
\end{aligned}
$$

in which $e_{m n}^{o}$ is given in 30 and $\bar{\Upsilon}_{m n}=$ $\frac{1}{L^{2}} \operatorname{tr}\left[\mathbf{P}_{m n} \overline{\mathbf{H}}_{m n}^{\mathrm{H}} \mathbf{C}_{m}^{-1} \boldsymbol{\Theta}_{m n} \mathbf{C}_{m}^{-1} \overline{\mathbf{H}}_{m n}\right]$, which can be written as

$$
\bar{\Upsilon}_{m n}=\frac{1}{L} \sum_{\substack{n^{\prime}=1 \\ n^{\prime} \neq n}}^{N} p_{m n^{\prime}} \overline{\mathbf{g}}_{m n^{\prime}}^{\mathrm{H}} \mathbf{\Theta}_{m n^{\prime}}^{1 / 2} \mathbf{C}_{m}^{-1} \Theta_{m n} \mathbf{C}_{m}^{-1} \boldsymbol{\Theta}_{m n^{\prime}}^{1 / 2} \overline{\mathbf{g}}_{m n^{\prime}}
$$

Based on Theorem 2 of [23], we have

$$
\bar{\Upsilon}_{m n} \stackrel{a . s .}{\rightarrow} \Upsilon_{m n}
$$

where $\Upsilon_{m n}$ is given in 33.
Inserting (79) and 80 in 55, we obtain

$$
\begin{gathered}
\sum_{\substack{n^{\prime}=1 \\
n^{\prime} \neq n}}^{N} p_{n^{\prime}} \sum_{m=1}^{M} \mathbb{E}\left\{\left|\mathbf{h}_{m n k}^{\mathrm{H}} \mathbf{w}_{m n^{\prime}}\right|^{2}\right\} \\
\stackrel{\text { a.s. }}{\rightarrow} \sum_{m=1}^{M} \Upsilon_{m n}\left(\frac{c_{m n k}^{2}}{\left(1+e_{m n}^{o}\right)^{2}}+a_{m n k}^{2}\right) .
\end{gathered}
$$

Finally, by substituting (68), (70), (71) and (83) in $(15), \gamma_{n k}^{\mathrm{mRZF}}$ is obtained as 29 .

\section{REFERENCES}

[1] M. Series, "IMT vision-framework and overall objectives of the future development of IMT for 2020 and beyond," 2015.

[2] M. Matthaiou, C. Zhong, M. R. McKay, and T. Ratnarajah, "Sum rate analysis of $\mathrm{ZF}$ receivers in distributed MIMO systems," IEEE J. Sel. Areas Commun., vol. 31, pp. 180-191, Feb. 2013.

[3] D. Wang, J. Wang, X. You, Y. Wang, M. Chen, and X. Hou, "Spectral efficiency of distributed MIMO systems," IEEE J. Sel. Areas Commun., vol. 31, pp. 2112 2127, Oct. 2013.

[4] C. He, J. Yin, Y. He, M. Huang, and B. Zhao, "Energy efficiency of distributed massive MIMO systems," Journal of Communications and Networks, vol. 18, pp. 649-657, Aug. 2016.

[5] Y. Huang, G. Zheng, M. Bengtsson, K. Wong, L. Yang, and B. Ottersten, "Distributed multicell beamforming with limited intercell coordination," IEEE Trans. on Signal Processing, vol. 59, pp. 728-738, Feb. 2011.

[6] J. Wang and L. Dai, "Asymptotic rate analysis of downlink multi-user systems with co-located and distributed antennas," IEEE Trans. Wireless Commun., vol. 14, pp. 3046-3058, June 2015.

[7] G. Interdonato, E. Björnson, P. Frenger, and E. G. Larsson, "Ubiquitous cell-free massive MIMO communications," 2019.

[8] H. Q. Ngo, A. Ashikhmin, H. Yang, E. G. Larsson, and T. L. Marzetta, "Cell-free massive MIMO versus small cells," IEEE Trans. Wireless Commun., vol. 16, pp. 1834 1850, Mar. 2017.

[9] E. Nayebi, A. Ashikhmin, T. L. Marzetta, H. Yang, and B. D. Rao, "Precoding and power optimization in cell-free massive MIMO systems," IEEE Trans. Wireless Commun., vol. 16, pp. 4445-4459, Jul. 2017.

[10] L. D. Nguyen, T. Q. Duong, H. Q. Ngo, and K. Tourki, "Energy efficiency in cell-free massive MIMO with zeroforcing precoding design," IEEE Commun. Lett., vol. 21, pp. 1871-1874, Aug. 2017.

[11] H. Q. Ngo, L. Tran, T. Q. Duong, M. Matthaiou, and E. G. Larsson, "On the total energy efficiency of cell-free massive MIMO," IEEE Trans. Green Commun. Netw., vol. 2, pp. 25-39, Mar. 2018.

[12] M. Attarifar, A. Abbasfar, and A. Lozano, "Modified conjugate beamforming for cell-free massive MIMO," IEEE Wireless Commun. Lett., vol. 8, pp. 616-619, April 2019. 


$$
\begin{aligned}
\mathbf{h}_{m n k}^{\mathrm{H}} \boldsymbol{\Sigma}_{m} \overline{\mathbf{H}}_{m n} \mathbf{P}_{m n} \overline{\mathbf{H}}_{m n}^{\mathrm{H}} \boldsymbol{\Sigma}_{m} \mathbf{h}_{m n k} & =\frac{1}{L} c_{m n k}^{2} \overline{\mathbf{g}}_{m n}^{\mathrm{H}} \boldsymbol{\Theta}_{m n}^{1 / 2} \mathbf{C}_{m n}^{-1} \overline{\mathbf{H}}_{m n} \mathbf{P}_{m n} \overline{\mathbf{H}}_{m n}^{\mathrm{H}} \mathbf{C}_{m}^{-1} \boldsymbol{\Theta}_{m n}^{1 / 2} \overline{\mathbf{g}}_{m n} \\
& +\frac{1}{L} c_{m n k}^{2} \overline{\mathbf{g}}_{m n}^{\mathrm{H}} \boldsymbol{\Theta}_{m n}^{1 / 2}\left(\mathbf{C}_{m}^{-1}-\mathbf{C}_{m n}^{-1}\right) \overline{\mathbf{H}}_{m n} \mathbf{P}_{m n} \overline{\mathbf{H}}_{m n}^{\mathrm{H}} \mathbf{C}_{m}^{-1} \boldsymbol{\Theta}_{m n}^{1 / 2} \overline{\mathbf{g}}_{m n} \\
& +\frac{1}{L} a_{m n k}^{2} \hat{\mathbf{g}}_{m n k}^{\mathrm{H}} \mathbf{\Theta}_{m n}^{1 / 2} \mathbf{C}_{m n}^{-1} \overline{\mathbf{H}}_{m n} \mathbf{P}_{m n} \overline{\mathbf{H}}_{m n}^{\mathrm{H}} \mathbf{C}_{m}^{-1} \mathbf{\Theta}_{m n}^{1 / 2} \hat{\mathbf{g}}_{m n k} \\
& +\frac{1}{L} a_{m n k}^{2} \hat{\mathbf{g}}_{m n k}^{\mathrm{H}} \boldsymbol{\Theta}_{m n}^{1 / 2}\left(\mathbf{C}_{m}^{-1}-\mathbf{C}_{m n}^{-1}\right) \overline{\mathbf{H}}_{m n} \mathbf{P}_{m n} \overline{\mathbf{H}}_{m n}^{\mathrm{H}} \mathbf{C}_{m}^{-1} \boldsymbol{\Theta}_{m n}^{1 / 2} \hat{\mathbf{g}}_{m n k} \\
& +\frac{1}{L} c_{m n k} a_{m n k} \overline{\mathbf{g}}_{m n}^{\mathrm{H}} \boldsymbol{\Theta}_{m n}^{1 / 2} \mathbf{C}_{m n}^{-1} \overline{\mathbf{H}}_{m n} \mathbf{P}_{m n} \overline{\mathbf{H}}_{m n}^{\mathrm{H}} \mathbf{C}_{m}^{-1} \mathbf{\Theta}_{m n}^{1 / 2} \hat{\mathbf{g}}_{m n k} \\
& +\frac{1}{L} c_{m n k} a_{m n k} \overline{\mathbf{g}}_{m n}^{\mathrm{H}} \boldsymbol{\Theta}_{m n}^{1 / 2}\left(\mathbf{C}_{m}^{-1}-\mathbf{C}_{m n}^{-1}\right) \overline{\mathbf{H}}_{m n} \mathbf{P}_{m n} \overline{\mathbf{H}}_{m n}^{\mathrm{H}} \mathbf{C}_{m}^{-1} \boldsymbol{\Theta}_{m n}^{1 / 2} \hat{\mathbf{g}}_{m n k} \\
& +\frac{1}{L} c_{m n k} a_{m n k} \hat{\mathbf{g}}_{m n k}^{\mathrm{H}} \mathbf{\Theta}_{m n}^{1 / 2} \mathbf{C}_{m n}^{-1} \overline{\mathbf{H}}_{m n} \mathbf{P}_{m n} \overline{\mathbf{H}}_{m n}^{\mathrm{H}} \mathbf{C}_{m}^{-1} \mathbf{\Theta}_{m n}^{1 / 2} \overline{\mathbf{g}}_{m n} \\
& +\frac{1}{L} c_{m n k} a_{m n k} \hat{\mathbf{g}}_{m n k}^{\mathrm{H}} \boldsymbol{\Theta}_{m n}^{1 / 2}\left(\mathbf{C}_{m}^{-1}-\mathbf{C}_{m n}^{-1}\right) \overline{\mathbf{H}}_{m n} \mathbf{P}_{m n} \overline{\mathbf{H}}_{m n}^{\mathrm{H}} \mathbf{C}_{m}^{-1} \boldsymbol{\Theta}_{m n}^{1 / 2} \overline{\mathbf{g}}_{m n}
\end{aligned}
$$

$$
\begin{aligned}
\mathbf{h}_{m n k}^{\mathrm{H}} \boldsymbol{\Sigma}_{m} \overline{\mathbf{H}}_{m n} \mathbf{P}_{m n} \overline{\mathbf{H}}_{m n}^{\mathrm{H}} \boldsymbol{\Sigma}_{m} \mathbf{h}_{m n k} & =c_{m n k}^{2}\left(\frac{1}{L} \overline{\mathbf{g}}_{m n}^{\mathrm{H}} \mathbf{B}_{m n} \overline{\mathbf{g}}_{m n}-\frac{1}{L} \overline{\mathbf{g}}_{m n}^{\mathrm{H}} \mathbf{A}_{m n} \overline{\mathbf{g}}_{m n} \overline{\mathbf{g}}_{m n}^{\mathrm{H}} \mathbf{B}_{m n} \overline{\mathbf{g}}_{m n}\right) \\
& +a_{m n k}^{2}\left(\frac{1}{L} \hat{\mathbf{g}}_{m n k}^{\mathrm{H}} \mathbf{B}_{m n} \hat{\mathbf{g}}_{m n k}-\frac{1}{L} \hat{\mathbf{g}}_{m n k}^{\mathrm{H}} \mathbf{A}_{m n} \overline{\mathbf{g}}_{m n} \overline{\mathbf{g}}_{m n}^{\mathrm{H}} \mathbf{B}_{m n} \hat{\mathbf{g}}_{m n k}\right) \\
& +a_{m n k} c_{m n k}\left(\frac{1}{L} \overline{\mathbf{g}}_{m n}^{\mathrm{H}} \mathbf{B}_{m n} \hat{\mathbf{g}}_{m n k}-\frac{1}{L} \overline{\mathbf{g}}_{m n}^{\mathrm{H}} \mathbf{A}_{m n} \overline{\mathbf{g}}_{m n} \overline{\mathbf{g}}_{m n}^{\mathrm{H}} \mathbf{B}_{m n} \hat{\mathbf{g}}_{m n k}\right) \\
& +a_{m n k} c_{m n k}\left(\frac{1}{L} \hat{\mathbf{g}}_{m n k}^{\mathrm{H}} \mathbf{B}_{m n} \overline{\mathbf{g}}_{m n}-\frac{1}{L} \hat{\mathbf{g}}_{m n k}^{\mathrm{H}} \mathbf{A}_{m n} \overline{\mathbf{g}}_{m n} \overline{\mathbf{g}}_{m n}^{\mathrm{H}} \mathbf{B}_{m n} \overline{\mathbf{g}}_{m n}\right)
\end{aligned}
$$

[13] T. H. Nguyen, T. K. Nguyen, H. D. Han, and V. D. Nguyen, "Optimal power control and load balancing for uplink cell-free multi-user massive MIMO," IEEE Access, vol. 6, pp. 14462-14473, 2018.

[14] J. Zhang, Y. Wei, E. Björnson, Y. Han, and S. Jin, "Performance analysis and power control of cell-free massive MIMO systems with hardware impairments," IEEE Access, vol. 6, pp. 55302-55314, 2018.

[15] S. M. R. Islam, N. Avazov, O. A. Dobre, and K. Kwak, "Power-domain non-orthogonal multiple access (NOMA) in 5G systems: Potentials and challenges," IEEE Commun. Surveys Tuts., vol. 19, pp. 721-742, Secondquarter 2017.

[16] S. Ali, E. Hossain, and D. I. Kim, "Non-orthogonal multiple access (NOMA) for downlink multiuser MIMO systems: User clustering, beamforming, and power allocation," IEEE Access, vol. 5, pp. 565-577, 2017.

[17] X. Chen, F. Gong, G. Li, H. Zhang, and P. Song, "User pairing and pair scheduling in massive MIMO-NOMA systems," IEEE Commun. Lett., vol. 22, pp. 788-791, Apr. 2018.

[18] D. Kudathanthirige and G. A. A. Baduge, "Noma-aided multicell downlink massive mimo," IEEE J. Sel. Topic Signal Processing, vol. 13, pp. 612-627, June. 2019.

[19] K. Senel, H. V. Cheng, E. Björnson, and E. G. Larsson, "What role can NOMA play in massive MIMO?," IEEE J. Sel. Topic Signal Processing., Sep. 2019, IEEE Xplore Early Access.

[20] Y. Li and G. A. Aruma Baduge, "NOMA-aided cell-free massive MIMO systems," IEEE Wireless Commun. Lett., vol. 7, pp. 950-953, Dec. 2018.

[21] Y. Li and G. A. Aruma Baduge, "NOMA-aided massive MIMO downlink with distributed antenna arrays," Proc.IEEE ICC, pp. 1-7, May. 2019.

[22] M. Bashar, K. Cumanan, G. Burr, H. Ngo, L.Hanzo, and P. Xiao, "NOMA/OMA mode selection-based cell-free massive MIMO," Proc.IEEE ICC, pp. 1-7, May. 2019.

[23] S. Wagner, R. Couillet, M. Debbah, and D. T. M. Slock, "Large system analysis of linear precoding in correlated MISO broadcast channels under limited feedback," IEEE Trans. Inf. Theory, vol. 58, pp. 4509-4537, July. 2012.

[24] H. Huh, A. M. Tulino, and G. Caire, "Network MIMO with linear zero-forcing beamforming: Large system analysis, impact of channel estimation, and reducedcomplexity scheduling," IEEE Trans. Inf. Theory, vol. 58, pp. 2911-2934, May. 2012.

[25] F. Rezaei and A. Tadaion, "Multi-layer beamforming in uplink/downlink massive MIMO systems with multiantenna users," Signal Processing, vol. 164, pp. 58-66, 2019.

[26] T. Van Chien, E. Björnson, and E. G. Larsson, "Joint power allocation and user association optimization for massive MIMO systems," IEEE Trans. Wireless Commun., vol. 15, pp. 6384-6399, Sep. 2016.

[27] G. Interdonato, M. Karlsson, E. Björnson, and E. G. Larsson, "Downlink spectral efficiency of cell-free massive MIMO with full-pilot zero-forcing," in Proc. IEEE Glob. Conf. Signal Inf. Process. (GlobalSIP), pp. 1003-1007, 
Nov. 2018.

[28] A. Benjebbour, A. Li, Y. Kishiyama, H. Jiang, and T. Nakamura, "System-level performance of downlink NOMA combined with SU-MIMO for future LTE enhancements," in Proc. Globecom Workshops (GC Wkshps), pp. 706-710, Dec. 2014.

[29] A. Benjebbour, Y. Saito, Y. Kishiyama, A. Li, A. Harada, and T. Nakamura, "Concept and practical considerations of non-orthogonal multiple access (NOMA) for future radio access," in Proc. Int. Symp. Intell. Signal Process. Commun. Syst., pp. 770-774, Nov. 2013.

[30] M. Zeng, A. Yadav, O. A. Dobre, G. I. Tsiropoulos, and H. V. Poor, "Capacity comparison between MIMONOMA and MIMO-OMA with multiple users in a cluster," IEEE J. Sel. Areas Commun., vol. 35, pp. 24132424, Oct. 2017.

[31] Z. Ding, F. Adachi, and H. V. Poor, "The application of MIMO to non-orthogonal multiple access," IEEE Trans. Wireless Commun., vol. 15, pp. 537-552, Jan. 2016.

[32] Y. Huang, C. Zhang, J. Wang, Y. Jing, L. Yang, and X. You, "Signal processing for MIMO-NOMA: Present and future challenges," IEEE Wireless Commun., vol. 25, pp. 32-38, Apr. 2018.

[33] M. Vaezi, G. Amarasuriya, Y. Liu, A. Arafa, F. Fang, and Z. Ding, "Interplay between NOMA and other emerging technologies: A survey," arXiv:1903.10489., Mar. 2019.

[34] Z. Ding and H. V. Poor, "Design of massive-MIMONOMA with limited feedback," IEEE Signal Process. Lett., vol. 23, pp. 629-633, May. 2016.

[35] D. Tse and P. Viswanath, Fundamentals of Wireless Communication. Cambridge University Press, 2005.

[36] Z. Chen and E. Björnson, "Channel hardening and favorable propagation in cell-free massive MIMO with stochastic geometry," IEEE Trans. Commun., vol. 66, pp. 5205-5219, Nov. 2018.

[37] S. M. Kay, Fundamentals of Statistical Signal Processing: Estimation Theory. Englewood Cliffs, NJ, USA: Prentice-Hall, 1993.

[38] P. Li, R. C. de Lamare, and R. Fa, "Multiple feedback successive interference cancellation detection for multiuser MIMO systems," IEEE Trans. Wireless Commun., vol. 10, pp. 2434-2439, Aug. 2011.

[39] T. L. Marzetta, E. G. Larsson, H. Yang, and H. Q. Ngo, Fundamentals of Massive MIMO. Cambridge: Cambridge University Press, 2016.

[40] A. M. Tulino and S. Verdu, Random Matrix Theory and Wireless Communications. Found. Trends Commun. Inf. Theory, 2004.

[41] J. Hoydis, S. ten Brink, and M. Debbah, "Massive MIMO in the UL/DL of cellular networks: How many antennas do we need?," IEEE J. Sel. Areas Commun., vol. 31, pp. 160-171, Feb. 2013.

[42] A. Tang, J. Sun, and K. Gongand, "Mobile propagation loss with a low base station antenna for NLOS street microcells in urban area," in Proc. IEEE Veh. Technol. Conf. (VTC), pp. 333-336, May. 2001.

[43] M. F. Hanif, Z. Ding, T. Ratnarajah, and G. K. Karagiannidis, "A minorization-maximization method for optimiz- ing sum rate in the downlink of non-orthogonal multiple access systems," IEEE Trans. on Signal Processing, vol. 64, pp. 76-88, Jan. 2016.

[44] J. Zhu, R. Schober, and V. K. Bhargava, "Linear precoding of data and artificial noise in secure massive MIMO systems," IEEE Trans. Wireless Commun., vol. 15, pp. 2245-2261, March. 2016.

[45] R. Couillet and M. Debbah, Random Matrix Methods for Wireless Communications. 1st ed. New York, NY, USA: Cambridge University Press, 2011. 